\title{
A transport formulation for moving fronts and application to dislocation dynamics
}

\author{
R. MONnEAU ${ }^{\dagger}$ \\ CERMICS, École nationale des Ponts et Chaussées, \\ 6 et 8 avenue Blaise Pascal, Cité Descartes, \\ Champs-sur-Marne, 77455 Marne-la-Vallée Cedex 2, France
}

[Received 29 May 2006 and in revised form 9 February 2007]

\begin{abstract}
We consider hypersurfaces moving with normal velocity depending on the time-space coordinates and on the normal to the hypersurface. We naturally define a measure associated to this hypersurface. This measure is defined on a suitable space/unit normal/curvature configuration space. We show that, while the hypersurface stays smooth, the measure is a solution to a linear transport equation, which we call a transport formulation. In the particular case of curves moving in the plane, we get a simple transport formulation. With this transport formulation in hand, it is then easy to complete the models of dislocation densities that were proposed in the 60's. As a consequence, we propose a closed mean field model for the dynamics of dislocation densities.
\end{abstract}

2000 Mathematics Subject Classification: 35R35, 35F20.

Keywords: kinetic equation, Liouville equation, transport equation, fronts, normal velocity, curvature, dislocation dynamics, dislocation densities.

\section{Introduction}

\subsection{Motivation}

We are interested in the motion of a smooth bounded connected and oriented hypersurface $\Gamma_{t} \subset \mathbb{R}^{N}$ with the first order geometric motion given by the normal velocity

$$
V=c(t, y, n(t, y))
$$

where $t \in[0, T)$ and $y \in \mathbb{R}^{N}$ denote respectively the time and space coordinates, and $n(t, y) \in$ $\mathbb{S}^{N-1}$ denotes the unit normal to $\Gamma_{t}$ at the point $y$ (for a given choice of orientation). We denote by $K(t, y) \in \mathbb{R}_{\mathrm{sym}}^{N \times N}$ the curvature of $\Gamma_{t}$ at the point $y$. This curvature is a symmetric $N \times N$ matrix, and will be defined precisely later. For a given $T>0$, we define

$$
\Gamma=\bigcup_{t \in[0, T)}\{t\} \times \Gamma_{t} \subset[0, T) \times \mathbb{R}^{n}
$$

To avoid any problem of regularity in this article, we will assume that

$$
\left\{\begin{array}{l}
\Gamma \in C^{3} \text { and } \quad c \in C^{3}\left([0, T) \times \mathbb{R}^{N} \times \mathbb{S}^{N-1}\right), \\
\forall t \in[0, T), \quad \Gamma_{t} \text { is } C^{3} \text { bounded, oriented and connected. }
\end{array}\right.
$$

It is well-known that we cannot expect in general existence of smooth solutions $\Gamma_{t}$ for all time and that singularities may happen in finite time.

†E-mail: monneau@cermics.enpc.fr 
Our goal is to show that it is possible to provide a transport formulation of the motion of such fronts. Our motivation comes from the modelling of dislocation dynamics, i.e. in the dynamics of curves moving in their slip planes in crystals. Physically, it is interesting to be able to sum the evolution of several lines to deduce statistical and mean properties of this dynamics. The challenge behind this question is the possibility to describe the dynamics of densities of such curves. We refer to the work of Sedláček, Kratochvíl and Werner [27], which was a source of inspiration for the present article.

In this paper, we show that our first goal can be achieved, at least while the solution $\Gamma_{t}$ stays smooth. Indeed, we prove (see Theorem 4.1) that the "density"

$$
g(t, y, n, K)=\delta_{\Gamma_{t}}(y) \delta_{0}(n-n(t, y)) \delta_{0}(K-K(t, y)),
$$

which is a measure for $(t, y, n, K) \in[0, T) \times \mathbb{R}^{N} \times \mathbb{S}^{N-1} \times \mathbb{R}_{\text {sym }}^{N \times N}$, satisfies the equation

$$
g_{t}+\operatorname{div}(a g)+a_{0} g=0,
$$

which is a linear transport equation for some function $a_{0}$ and a suitable vector field $a$ (which is related to characteristics of Hamilton-Jacobi equations). The precise meaning and the details of these expressions will be given later (see Subsection 1.3 and Theorem 4.1). Let us mention that the vector field $a$ has a quadratic growth at infinity, as a function of the curvature $K$. This naturally creates some mathematical difficulties (which will not be addressed in the present paper) in getting long time existence of solutions. This is obviously related to the fact that geometrically, the curvature of $\Gamma_{t}$ can become infinite in finite time.

We were tempted to call the equation satisfied by $g$ a "kinetic formulation", but this terminology has already been used to denote a powerful approach to nonlinear hyperbolic equations (see Perthame [26] and the references therein). This approach allows one to get existence and uniqueness results, even after the appearance of singularities of the solution. On the contrary, our transport formulation only deals with smooth evolutions (even if it would be interesting to extend it after the appearance of singularities). Let us also cite a related famous example of transport equation associated to nonlinear evolution: this is the hamiltonian formulation of the Euler equation by Oseledets [24].

After our work was completed, we have become aware of the results by Hochrainer and Zaiser [13] (see also Hochrainer [12] and Zaiser and Hochrainer [31]) that seem similar in the special case of dimension $N=2$ for velocities $c(t, y)$ independent of the normal $n$, these results being based on the notion of Lie derivative of differential forms.

Our work focuses on transport formulations for hypersurfaces. In the appendix, we only give some indications on how to get other transport formulations associated to the wavefront of the evolution of submanifolds of codimension higher than 1, in particular for the case of the transport of curves in $\mathbb{R}^{N}$.

In the particular case of curves moving in the plane, it is possible to use a simplified description. We can describe the normal $n=(\cos \theta, \sin \theta)$ by its angle $\theta \in \mathbb{R} /(2 \pi \mathbb{Z})$ and choose a scalar curvature $\kappa \in \mathbb{R}$. In this framework a transport formulation (see Theorem 2.1) is proposed for the "density"

$$
g(t, y, \theta, \kappa)=\delta_{\Gamma_{t}}(y) \delta_{0}(\theta-\theta(t, y)) \delta_{0}(\kappa-\kappa(t, y)) .
$$

Finally, let us mention that our analysis does not cover the case of velocities depending on the curvature itself. This would be an interesting extension in connection with random processes (see 
for instance Buckdahn, Cardaliaguet and Quincampoix [3], Soner and Touzi [28-30]). We plan to study this problem in a future work.

\subsection{Organization of the paper}

In Section 2, we present our main result in dimension $N=2$, namely Theorem 2.1 for the simplified description. As an application, we also propose a model for the dynamics of dislocation densities (see Subsection 2.2). In Section 3, we give the proofs of Theorem 2.1 and of Proposition 2.2.

For completeness, we state in Section4 4 our main result in any dimension $N \geqslant 2$ (Theorem 4.1). The proof is basically similar to the one of Theorem 2.1. but technically more involved. This is the reason why we have chosen to present the result in general dimension after the result in dimension $N=2$. The proof of Theorem 4.1 is given in Section 5 . In the appendix, for completeness, we give in Subsection 6.1 the proof of Lemma 5.1 in Subsection 6.2 we give some indications about a transport formulation of the motion of curves in $\mathbb{R}^{N}$ in the case of pure transport; and in Subsection 6.3. we propose an alternative transport equation for the wavefront of curves moving in the plane which is well-posed for long time existence of solutions.

\subsection{Notation}

For a smooth oriented hypersurface $\Gamma_{t}$ in $\mathbb{R}^{N}$, we denote by $n(t, y) \in \mathbb{S}^{N-1}$ the unit normal to $\Gamma_{t}$ at the point $y \in \Gamma_{t}$, and by $K(t, y) \in \mathbb{R}_{\mathrm{sym}}^{N \times N}$ its curvature, where $\mathbb{R}_{\mathrm{sym}}^{N \times N}$ is the set of symmetric $N \times N$ matrices. This matrix $K(t, y)$ is given by

$$
K(t, y)=\sum_{i=1}^{N-1} K_{i} f_{i} \otimes f_{i}
$$

where the $K_{i}$ are the principal curvatures and the $f_{i}$ are the principal directions of curvature of the surface $\Gamma_{t}$ at the point $y$. Recall that the $f_{i}, i=1, \ldots, N-1$, generate the tangent hyperplane to $\Gamma_{t}$ at the point $y$. We use here the convention that for a sphere, if the normal is pointing outwards from the ball, then the curvatures $K_{i}$ are negative.

In dimension $N=2$, we set

$$
n=(\cos \theta, \sin \theta), \quad \tau=(\sin \theta,-\cos \theta),
$$

where $(\tau, n)$ is a positively oriented orthonormal basis. Depending on the context (but without ambiguity) we will consider either general $\tau, n$ depending on the general variable $\theta \in \mathbb{R} /(2 \pi \mathbb{Z})$ (and sometimes denoted by $\tau(\theta), n(\theta)$ to clearly specify the dependence on $\theta$ ), or depending on the particular value $\theta(t, y) \in \mathbb{R} /(2 \pi \mathbb{Z})$ which is the angle associated to $n(t, y)$ for $y \in \Gamma_{t}$. We will also define the scalar curvature $\kappa(t, y)$ by

$$
K(t, y)=\kappa(t, y) \tau \otimes \tau .
$$

We denote by $\partial_{\theta}$ and $\partial_{\kappa}$ the derivatives respectively with respect to $\theta$ and to $\kappa$. With this notation, we have in particular

$$
\partial_{\theta} \tau=n, \quad \partial_{\theta} n=-\tau
$$


In any dimension $N$, considering a positively oriented orthonormal basis $\left(e_{1}, \ldots, e_{N}\right)$, and for tensors $\mathcal{T}=\sum_{i_{1}, \ldots, i_{T}=1}^{N} \mathcal{T}_{i_{1}, \ldots, i_{T}} e_{i_{1}} \otimes \cdots \otimes e_{i_{T}}, \mathcal{S}=\sum_{j_{1}, \ldots, j_{T}=1}^{N} \mathcal{S}_{j_{1}, \ldots, j_{S}} e_{j_{1}} \otimes \cdots \otimes e_{j_{S}}$, we define the simple contraction of tensors

$$
\mathcal{T} \cdot \mathcal{S}=\sum_{i_{1}, \ldots, i_{T-1}=1}^{N} \sum_{j_{2}, \ldots, j_{S}=1}^{N}\left(\sum_{k=1}^{N} \mathcal{T}_{i_{1}, \ldots, i_{T-1}, k} \mathcal{S}_{k, j_{2}, \ldots, j_{S}}\right) e_{i_{1}} \otimes \cdots \otimes e_{i_{T-1}} \otimes e_{j_{2}} \otimes \cdots \otimes e_{j_{S}}
$$

and the double contraction of tensors

$$
\mathcal{T}: \mathcal{S}=\sum_{i_{1}, \ldots, i_{T-2}=1}^{N} \sum_{j_{3}, \ldots, j_{S}=1}^{N}\left(\sum_{k, l=1}^{N} \mathcal{T}_{i_{1}, \ldots, i_{T-2}, l, k} \mathcal{S}_{k, l, j_{3}, \ldots, j_{S}}\right) e_{i_{1}} \otimes \cdots \otimes e_{i_{T-2}} \otimes e_{j_{3}} \otimes \cdots \otimes e_{j_{S}}
$$

Assuming that the tensor $\mathcal{T}$ depends on $K \in \mathbb{R}_{\text {sym }}^{N \times N}$, we define

$$
\partial_{K} \mathcal{T}=\sum_{p, q=1}^{N} \frac{1}{2}\left(1+\delta_{p q}\right) \sum_{i_{1}, \ldots, i_{T}=1}^{N}\left(\frac{\partial}{\partial K_{p q}} \mathcal{T}_{i_{1}, \ldots, i_{T}}\right) e_{p} \otimes e_{q} \otimes e_{i_{1}} \otimes \cdots \otimes e_{i_{T}} .
$$

With this definition, we have for instance $\mathcal{T}: \partial_{K} K=\mathcal{T}$ if $\mathcal{T}$ is symmetric in its last two indices. Similarly, for a tensor $\mathcal{T}$ depending on $y \in \mathbb{R}^{N}$, we define

$$
\partial_{y} \mathcal{T}=\sum_{p=1}^{N} \sum_{i_{1}, \ldots, i_{T}=1}^{N}\left(\frac{\partial}{\partial y_{p}} \mathcal{T}_{i_{1}, \ldots, i_{T}}\right) e_{p} \otimes e_{i_{1}} \otimes \cdots \otimes e_{i_{T}}
$$

Similarly, if the tensor $\mathcal{T}(n)$ depends on $n \in \mathbb{S}^{N-1}$ (among other possible variables), we consider $\tilde{n}$ in a neighbourhood of $\mathbb{S}^{N-1}$ in $\mathbb{R}^{N}$ and then define $\widetilde{\mathcal{T}}_{i_{1}, \ldots, i_{T}}(\tilde{n})=\mathcal{T}_{i_{1}, \ldots, i_{T}}(\tilde{n} /|\tilde{n}|)$. We set

$$
\partial_{n} \mathcal{T}(n)=\sum_{p=1}^{N} \sum_{i_{1}, \ldots, i_{T}=1}^{N}\left(\frac{\partial}{\partial \tilde{n}_{p}}\left(\widetilde{\mathcal{T}}_{i_{1}, \ldots, i_{T}}(\tilde{n})\right)\right)_{\mid \tilde{n}=n} e_{p} \otimes e_{i_{1}} \otimes \cdots \otimes e_{i_{T}}
$$

We have in particular $n \cdot \partial_{n}=0$ and $\partial_{n} n=I-n \otimes n$ with the identity matrix $I=\sum_{i=1}^{N} e_{i} \otimes e_{i}$. We also define

$$
\partial_{y y}^{2} \mathcal{T}=\partial_{y}\left(\partial_{y} \mathcal{T}\right), \quad \partial_{y n}^{2} \mathcal{T}=\partial_{y}\left(\partial_{n} \mathcal{T}\right), \quad \partial_{n y}^{2} \mathcal{T}=\partial_{n}\left(\partial_{y} \mathcal{T}\right), \quad \partial_{n n}^{2} \mathcal{T}=\partial_{n}\left(\partial_{n} \mathcal{T}\right)-n \otimes \partial_{n} \mathcal{T},
$$

where we can check that $\partial_{n n}^{2} \mathcal{T}$ is symmetric in its first two indices. We also let $\partial_{t} \mathcal{T}$ be the tensor whose components are the time derivatives of the components of $\mathcal{T}$. We set

$$
D_{t}=\partial_{t}+c n \cdot \partial_{y}, \quad D_{\tau}=-(I-n \otimes n) \cdot \partial_{y}+K \cdot \partial_{n} .
$$

Finally, in dimension $N=2$, we keep the same notation for defining

$$
D_{\tau}=\tau \cdot \partial_{y}+\kappa \partial_{\theta}
$$

and write

$$
\partial_{y y}^{2} \cdot=\partial_{y}\left(\partial_{y} \cdot\right), \quad \partial_{y \theta}^{2} \cdot=\partial_{y}\left(\partial_{\theta} \cdot\right), \quad \partial_{\theta y}^{2} \cdot=\partial_{\theta}\left(\partial_{y} \cdot\right), \quad \partial_{\theta \theta}^{2} \cdot=\partial_{\theta}\left(\partial_{\theta} \cdot\right) .
$$

For a function $f$, we also set

$$
\partial_{y}^{\perp} f=-\left(\frac{\partial f}{\partial y_{2}}\right) e_{1}+\left(\frac{\partial f}{\partial y_{1}}\right) e_{2} .
$$


2. Result in dimension $N=2$

\subsection{Main result}

In dimension $N=2$, let us consider a closed connected and oriented curve $\Gamma_{t}$ for $t \in[0, T)$ for some fixed $T>0$, with the normal pointing outwards from the bounded set whose boundary is the curve. At a point $y$ of the curve, we can write the unit normal $n(t, y)=(\cos \theta(t, y), \sin \theta(t, y))$ with $\theta(t, y) \in \mathbb{R} /(2 \pi \mathbb{Z})$, and we denote by $\kappa(t, y) \in \mathbb{R}$ the curvature (negative for a circle).

We set

$$
\bar{c}(t, y, \theta)=c(t, y, n(\theta))
$$

by abuse of notation, we will continue to denote it $c(t, y, \theta)$.

Then for any function $\varphi \in C_{c}^{\infty}\left([0, T) \times \mathbb{R}^{2} \times(\mathbb{R} /(2 \pi \mathbb{Z})) \times \mathbb{R}\right)$, we define the distribution $g_{\Gamma}(t, y, \theta, \kappa)$ by

$$
\left\langle g_{\Gamma}, \varphi\right\rangle=\int_{0}^{T} \mathrm{~d} t \int_{\Gamma_{t}} \varphi(t, y, \theta(t, y), \kappa(t, y)) .
$$

Given any distribution $g$ (with compact support in the variable $\kappa \in \mathbb{R}$ ), we also define formally the distribution $\hat{g}(t, y, \theta)$ by

$$
\hat{g}:=\int_{\mathbb{R}} \mathrm{d} \kappa D_{\tau} g \quad \text { with } \quad D_{\tau}=\tau \cdot \partial_{y}+\kappa \partial_{\theta}
$$

where $\tau$ is defined in $\left[1.3\right.$, i.e. rigorously, for any $\psi \in C_{c}^{\infty}\left([0, T) \times \mathbb{R}^{2} \times(\mathbb{R} /(2 \pi \mathbb{Z}))\right)$,

$$
\langle\hat{g}, \psi\rangle:=\left\langle D_{\tau} g, \bar{\psi}\right\rangle \quad \text { with } \quad \bar{\psi}(t, y, \theta, \kappa)=\psi(t, y, \theta) .
$$

Then we have the following result:

THEOREM 2.1 (Equivalence geometric motion/linear transport, $N=2$ ) Under the regularity assumption (1.2), if $\left(\Gamma_{t}\right)_{t}$ solves equation $(1.1)$ on the time interval $[0, T)$, then the distribution $g_{\Gamma}(t, y, \theta, \kappa)$ defined by (2.4) solves the equation

$$
g_{t}+\operatorname{div}(a g)+a_{0} g=0 \quad \text { in } \mathcal{D}^{\prime}\left((0, T) \times \mathbb{R}^{2} \times(\mathbb{R} /(2 \pi \mathbb{Z})) \times \mathbb{R}\right)
$$

with

$$
\operatorname{div}(a g)=\partial_{y} \cdot\left(a_{y} g\right)+\partial_{\theta}\left(a_{\theta} g\right)+\partial_{\kappa}\left(a_{\kappa} g\right) \quad \text { for } a=\left(a_{y}, a_{\theta}, a_{\kappa}\right)
$$

and

$$
\left\{\begin{array}{l}
a_{0}=\kappa\left(c+\partial_{\theta \theta}^{2} c\right)+\tau \cdot \partial_{y \theta}^{2} c, \quad a_{y}=c n-\tau \partial_{\theta} c, \quad a_{\theta}=\tau \cdot \partial_{y} c, \\
a_{\kappa}=\kappa^{2}\left(c+\partial_{\theta \theta}^{2} c\right)+\kappa\left(n \cdot \partial_{y} c+2 \tau \cdot \partial_{y \theta}^{2} c\right)+\tau \otimes \tau: \partial_{y y}^{2} c .
\end{array}\right.
$$

Moreover, if $g$ (with compact support in the variable $\kappa \in \mathbb{R}$ ) satisfies equation (2.6), then $\hat{g}$ defined by (2.5) satisfies the equation

$$
\hat{g}_{t}+\operatorname{div}\left(a^{\prime} \hat{g}\right)=0 \quad \text { in } \mathcal{D}^{\prime}\left((0, T) \times \mathbb{R}^{2} \times(\mathbb{R} /(2 \pi \mathbb{Z}))\right)
$$

with

$$
\operatorname{div}\left(a^{\prime} \hat{g}\right)=\partial_{y} \cdot\left(a_{y} \hat{g}\right)+\partial_{\theta}\left(a_{\theta} \hat{g}\right) \quad \text { for } a^{\prime}=\left(a_{y}, a_{\theta}\right) .
$$

Finally, for $g_{\Gamma}$ defined by 2.4, $\hat{g}_{\Gamma}$ defined in 2.5 satisfies $\hat{g}_{\Gamma}=0$. 
We note that a single planar curve is now represented as a measure on a space of dimension 4 , and this measure satisfies the linear transport equation (2.6). Note also that for a curve we necessarily have $\hat{g}_{\Gamma}=0$, which can be interpreted as a kind of compatibility condition. Moreover, this compatibility condition is preserved by the equation on $g$, because $\hat{g}$ satisfies equation [2.9].

Here, general distributions $g$ can be interpreted as the density of curves in the generalized space of space/angle/curvature coordinates. We do not know if in some sense, any distribution $g$ solving equation 2.6 and satisfying $\hat{g}=0$ can be written as a linear combination of measures $g_{\Gamma}$ for a possibly infinite number of evolutions $\Gamma$.

We easily see by an integration in $\theta$ that $\hat{g}=0$ implies (at least formally) that

$$
\partial_{y} \cdot\left(\int_{\mathbb{R} /(2 \pi \mathbb{Z}) \times \mathbb{R}} \tau(\theta) g(t, y, \theta, \kappa) \mathrm{d} \theta \mathrm{d} \kappa\right)=0,
$$

which can be interpreted as a conservation equation, namely the conservation of the Burgers vector along the dislocation lines, in the terminology of dislocation dynamics (see Lardner [19]). From a physical point of view (see [2]), the Burgers vector of a dislocation line is an invariant associated to the underlying lattice crystal. Mathematically this can be interpreted as the fact that the dislocation line has to be a closed loop.

More precisely, we have the following result:

PROPOSITION 2.2 (Transport equation for the vectorial density) If $g$ is a solution of (2.6) on the time interval $(0, T)$, then the vectorial distribution $\tau g$ satisfies, in $\left(\mathcal{D}^{\prime}\left((0, T) \times \mathbb{R}^{2} \times(\mathbb{R} /(2 \pi \mathbb{Z}))\right.\right.$ $\times \mathbb{R}))^{2}$,

$$
0=(\tau g)_{t}-\partial_{y}^{\perp}(c g)+\left(D_{\tau} g\right) a_{y}+\partial_{\theta}\left(\left(D_{\tau} c\right) \tau g-c \kappa n g\right)+\partial_{\kappa}\left(a_{\kappa} \tau g\right) .
$$

Equation 2.12 together with the assumption $\hat{g}=0$ shows in particular, by an integration in $\theta$ and $\kappa$, that we have (at least formally) the following evolution equation for the reduced vectorial density:

$$
\frac{\partial}{\partial t}\left(\int_{\mathbb{R} /(2 \pi \mathbb{Z}) \times \mathbb{R}} \tau(\theta) g(t, y, \theta, \kappa) \mathrm{d} \theta \mathrm{d} \kappa\right)=\partial_{y}^{\perp}\left(\int_{\mathbb{R} /(2 \pi \mathbb{Z}) \times \mathbb{R}} c(t, y, \theta) g(t, y, \theta, \kappa) \mathrm{d} \theta \mathrm{d} \kappa\right),
$$

which clearly preserves the divergence free property 2.11 .

Let us mention the mathematical difficulty due to the fact that the vector field $a$ has a quadratic growth in the curvature $\kappa$, which only allows one to expect short time existence of solutions to the transport equation in general. See the appendix (Subsection 6.3) for a different possible transport equation which overcomes this difficulty, and well describes the wavefront solution.

REMARK 2.3 After this work was completed, T. Hochrainer pointed out to me the following important observation (see Hochrainer, Zaiser and Gumbsch [14]). For any distribution $g$ satisfying 2.6) with compatibility condition $\hat{g}=0$, we can define

$$
\overline{\bar{g}}(t, y, \theta)=\int_{\mathbb{R}} \mathrm{d} \kappa g(t, y, \theta, \kappa), \quad \overline{\bar{\kappa}}(t, y, \theta)=\int_{\mathbb{R}} \mathrm{d} \kappa \kappa g(t, y, \theta, \kappa) .
$$

Then we get the compatibility condition

$$
\tau \cdot \partial_{y} \overline{\bar{g}}+\partial_{\theta} \overline{\bar{\kappa}}=0
$$


and (using integration by parts) the system

$$
\left\{\begin{array}{l}
\overline{\bar{g}}_{t}+\operatorname{div}\left(a^{\prime} \overline{\bar{g}}\right)+\left(\tau \cdot \partial_{y \theta}^{2} c\right) \overline{\bar{g}}+\left(c+\partial_{\theta \theta}^{2} c\right) \overline{\bar{\kappa}}=0 \\
\overline{\bar{\kappa}}_{t}+\operatorname{div}\left(a^{\prime} \overline{\bar{\kappa}}\right)-\left(n \cdot \partial_{y} c+\tau \cdot \partial_{y \theta}^{2} c\right) \overline{\bar{\kappa}}-\left(\tau \otimes \tau: \partial_{y y}^{2} c\right) \overline{\bar{g}}=0
\end{array}\right.
$$

with $a^{\prime}$ defined in 2.10). A remarkable property of the linear system 2.14 is that the coefficients are bounded, which ensures the existence of a solution $(\overline{\bar{g}}, \overline{\bar{\kappa}})$ for all time.

\subsection{Application to dislocation dynamics}

As an application, let us give a natural model for the dynamics of dislocation densities, using our transport formulation.

To simplify the presentation, let us consider only one slip system in a tridimensional crystal with orthonormal basis $\left(e_{1}, e_{2}, e_{3}\right)$, with dislocation curves moving in planes perpendicular to $e_{3}$, and with Burgers vector $b \in \mathbb{R}^{3}$ (with $b \cdot e_{3}=0$ for mobile dislocations without climb). We assume that the density of such dislocations is represented by the quantity

$$
g(t, x, \theta, \kappa) \quad \text { with } \quad x=\left(x_{1}, x_{2}, x_{3}\right) \in \mathbb{R}^{3}, \theta \in \mathbb{R} /(2 \pi \mathbb{Z}), \kappa \in \mathbb{R} .
$$

The strain $e(t, x) \in \mathbb{R}_{\mathrm{sym}}^{3 \times 3}$ solves on $\mathbb{R}^{3}$ (see for instance Alvarez et al. [2]) the system

$$
\left\{\begin{array}{l}
\operatorname{div}(\Lambda: e)=0, \\
\operatorname{inc} e=\left(\operatorname{curl}_{\text {row }}(b \otimes \beta)\right)_{\text {sym }} \quad \text { with } \quad \beta(t, x)=\int_{\mathbb{R} /(2 \pi \mathbb{Z}) \times \mathbb{R}} \mathrm{d} \theta \mathrm{d} \kappa \tau g(t, x, \theta, \kappa),
\end{array}\right.
$$

where $\Lambda=\left(\Lambda_{i j k l}\right)_{i, j, k, l=1,2,3}$ is the fourth order tensor of elastic coefficients, and the operator inc $e$ is obtained by taking first the curl of the column vectors of the matrix $e$, and then the curl of the row vectors of the new matrix. The curl $\mathrm{r}_{\text {row }}$ is the curl of the row vectors of the matrix, and the subscript ()$_{\text {sym }}$ means that we consider the symmetric part of the matrix. The quantity $b \otimes \beta$ is called the Nye tensor of dislocation densities. Here we set

$$
\tau=(\sin \theta) e_{1}-(\cos \theta) e_{2}, \quad n=(\cos \theta) e_{1}+(\sin \theta) e_{2}
$$

The normal velocity of the dislocations is proportional to the resolved Peach-Koehler force up to a drag coefficient. Even if it is easy (using equation 2.6) to write the model for an anisotropic drag coefficient, for simplicity let us restrict ourselves to the case where this coefficient is equal to 1 . Then the velocity is simply given by

$$
c(t, x)=\left(b \otimes e_{3}\right): e(t, x)
$$

and for $x=\left(y, x_{3}\right)$ with $y=\left(x_{1}, x_{2}\right)$, for each $x_{3} \in \mathbb{R}, g^{x_{3}}(t, y, \theta, \kappa):=g\left(t, y, x_{3}, \theta, \kappa\right)$ solves equation 2.6 with a normal velocity independent of $\theta$ defined by $c^{x_{3}}(t, y, \theta)=c\left(t, y\right.$, $\left.x_{3}\right)$, i.e.

$$
\begin{aligned}
0= & g_{t}^{x_{3}}+\kappa c^{x_{3}} g^{x_{3}}+n \cdot \partial_{y}\left(c^{x_{3}} g^{x_{3}}\right) \\
& +\partial_{y} c^{x_{3}} \cdot \partial_{\theta}\left(\tau g^{x_{3}}\right)+\partial_{\kappa}\left(g^{x_{3}}\left(c^{x_{3}} \kappa^{2}+\kappa n \cdot \partial_{y} c^{x_{3}}+\tau \otimes \tau: \partial_{y y}^{2} c^{x_{3}}\right)\right)
\end{aligned}
$$

i.e.

$$
0=g_{t}+\kappa c g+n \cdot \partial_{x}(c g)+\partial_{x} c \cdot \partial_{\theta}(\tau g)+\partial_{\kappa}\left(g\left(c \kappa^{2}+\kappa n \cdot \partial_{x} c+\tau \otimes \tau: \partial_{x x}^{2} c\right)\right) .
$$


The complete system of equations satisfied by $g$ is then $(2.15)-\sqrt{2.16}-(2.17)-(2.19)$, with a choice of the initial data satisfying the compatibility condition $\hat{g}=0$ in the notation $(2.5)$, i.e.

$$
\int_{\mathbb{R}} \mathrm{d} \kappa\left\{\tau \cdot \partial_{x} g+\kappa \partial_{\theta} g\right\}=0 .
$$

This system is a generalization of the model of Groma and Balogh [10, 11] that was restricted to the motion of straight line dislocations with curvature $\kappa=0$ and only two possible angles $\theta=0$ or $\pi$. See for instance El Hajj and Forcadel [8] for a mathematical analysis of the Groma-Balogh model in a particular geometry. In equation 2.19, the term $\kappa c g$ can be interpreted as a source term created by the curvatures of the dislocations. Our model is also a natural generalization of the model of Sedláček, Kratochvíl and Werner [27] whose transport equation was written for $\beta\left(t, y, x_{3}\right)$, namely

$$
\beta_{t}=\partial_{y}^{\perp}(c|\beta|) .
$$

This equation has to be compared to our equation (2.13) which has more degrees of freedom, or even to (2.12) or (2.19). We also underline that equation (2.19) is a natural transport equation that was missing for instance in the theory of Kröner [17, 18] and was under investigation in the theories of Mura [23] or Kosevich [16].

In the case where there are several slip systems, the contributions of all slip systems must be summed on the right hand side of the equation giving inc $e$, and each density solves an evolution equation similar to (2.6) in its own slip plane direction with its corresponding velocity. The complete system will be studied in a future work.

Let us remark that our model (2.15)-2.16)-(2.17)- 2.19] only describes pure transport of dislocation lines in a quite rough mean field model. For instance we do not treat self-annihilation of dislocation lines, in contrast to the eikonal equation. We in fact describe only a kind of wavefront propagation (as in Osher et al. [25], see also the appendix of the present article). Moreover our mean field model is really a zero-order approximation. A more realistic model would also contain some short distance corrections similar to the homogenization problem studied in Imbert and Monneau [15]. In a more realistic model, other source or collision terms should be added to describe Frank-Read sources, annihilations of dislocations, cross-slip, etc. See for instance the approach of El-Azab [7, 6].

\section{Proofs in dimension $N=2$}

We start with the following result:

Lemma 3.1 Let $\psi \in C_{c}^{1}\left((0, T) \times \mathbb{R}^{2}\right)$ and $\left(\Gamma_{t}\right)_{t}$ be a smooth evolution with normal velocity $c(t, y)$. Then

$$
\frac{\mathrm{d}}{\mathrm{d} t}\left(\int_{\Gamma_{t}} \psi\right)=\int_{\Gamma_{t}} D_{t} \psi-c \kappa \psi \quad \text { with } \quad D_{t} \psi:=\psi_{t}+c n \cdot \partial_{y} \psi
$$

where $\psi, n, \kappa$ and $c$ are evaluated at the current point $(t, y)$.

Proof. We fix the time $t$, and consider a parametrization $\gamma$ of the connected curve $\Gamma_{t}$ by the curvilinear abscissa $s$; the point $\gamma(s)$ is associated to $s \in \mathbb{R} /(L \mathbb{Z})$, with $L$ the length of $\Gamma_{t}$ and $\mathrm{d} \gamma / \mathrm{d} s=\tau$. Then we can parametrize the curve $\Gamma_{t+h}$ by $\gamma_{t+h}$ for $h$ small (even if the parametrization is not by curvilinear abscissa) where

$$
\gamma_{t+h}(s)=\gamma(s)+r(h, s) n(s)
$$


and $n(s)$ is the normal to $\Gamma_{t}$ at the point $\gamma(s)$. Moreover, $r(0, s)=0$ and $r_{h}(0, s)=c(t, \gamma(s))$ (where $r_{h}$ stands for $\partial r / \partial h$ and $r_{s}$ stands for $\partial r / \partial s$ ). We compute

$$
\begin{aligned}
\int_{\Gamma_{t+h}} \psi(t+h, \cdot) & =\int_{\mathbb{R} /(L \mathbb{Z})} \mathrm{d} s \psi\left(t+h, \gamma_{t+h}(s)\right)\left|\frac{\mathrm{d}}{\mathrm{d} s} \gamma_{t+h}(s)\right| \\
& =\int_{\mathbb{R} /(L \mathbb{Z})} \mathrm{d} s \psi(t+h, \gamma(s)+r(h, s) n)\left|(1-r(h, s) \kappa) \tau+r_{s}(h, s) n\right| .
\end{aligned}
$$

Therefore

$$
\begin{aligned}
\frac{\mathrm{d}}{\mathrm{d} h}\left(\int_{\Gamma_{t+h}} \psi(t+h, \cdot)\right)_{\mid h=0} & =\int_{\mathbb{R} /(L \mathbb{Z})} \mathrm{d} s\left\{\psi_{t}+r_{h}(0, s) n \cdot \partial_{y} \psi+\psi\left(-r_{h}(0, s) \kappa\right)\right\} \\
& =\int_{\Gamma_{t}}\left(\psi_{t}+c n \cdot \partial_{y} \psi-c \kappa \psi\right) .
\end{aligned}
$$

This ends the proof of the lemma.

LEMMA 3.2 For any $\varphi \in C_{c}^{\infty}\left((0, T) \times \mathbb{R}^{2} \times(\mathbb{R} /(2 \pi \mathbb{Z})) \times \mathbb{R}\right)$ and $g=g_{\Gamma}$, we have

$$
\left\langle g_{t}, \varphi\right\rangle=\left\langle g,-c \kappa \varphi+c n \cdot \partial_{y} \varphi+\left(D_{t} \tilde{\theta}\right) \partial_{\theta} \varphi+\left(D_{t} \tilde{\kappa}\right) \partial_{\kappa} \varphi\right\rangle
$$

for any $C^{1}$ extension $\tilde{\theta}$ (resp. $\left.\tilde{\kappa}\right)$ of $\theta$ (resp. $\left.\kappa\right)$.

Proof. We have

$$
\begin{aligned}
\left\langle g_{t}, \varphi\right\rangle & =-\left\langle g, \varphi_{t}\right\rangle=-\int_{0}^{T} \mathrm{~d} t \int_{\Gamma_{t}} \varphi_{t} \\
& =\int_{0}^{T} \mathrm{~d} t\left\{\frac{\mathrm{d}}{\mathrm{d} t}\left(\int_{\Gamma_{t}} \varphi\right)-\int_{\Gamma_{t}} \varphi_{t}\right\} .
\end{aligned}
$$

We now compute using 3.20 with $\psi(t, y)=\varphi(t, y, \tilde{\theta}(t, y), \tilde{\kappa}(t, y))$, and the velocity $c(t, y, \tilde{\theta}(t, y))$ :

$$
\begin{aligned}
\frac{\mathrm{d}}{\mathrm{d} t}\left(\int_{\Gamma_{t}} \varphi(t, y, \theta(t, y), \kappa(t, y))\right)= & \int_{\Gamma_{t}}\left(\varphi_{t}+\tilde{\theta}_{t} \partial_{\theta} \varphi+\tilde{\kappa}_{t} \partial_{\kappa} \varphi-c \kappa \varphi\right) \\
& +\int_{\Gamma_{t}} c n \cdot\left\{\partial_{y} \varphi+\left(\partial_{y} \tilde{\theta}\right) \partial_{\theta} \varphi+\left(\partial_{y} \tilde{\kappa}\right) \partial_{\kappa} \varphi\right\} .
\end{aligned}
$$

Then

$$
\left\langle g_{t}, \varphi\right\rangle=\int_{0}^{T} \mathrm{~d} t \int_{\Gamma_{t}}\left(-c \kappa \varphi+c n \cdot \partial_{y} \varphi+\left\{\tilde{\theta}_{t}+c n \cdot \partial_{y} \tilde{\theta}\right\} \partial_{\theta} \varphi+\left\{\tilde{\kappa}_{t}+c n \cdot \partial_{y} \tilde{\kappa}\right\} \partial_{\kappa} \varphi\right),
$$

which gives the result.

LEMMA 3.3 With the notation of Lemma 3.2, on $\Gamma$ we have

$$
\left\{\begin{array}{l}
D_{t} \tilde{\theta}=D_{\tau} c \\
D_{t} \tilde{\kappa}=a_{\kappa}+\left(\partial_{\theta} c\right)\left(\tau \cdot \partial_{y} \tilde{\kappa}\right)
\end{array}\right.
$$

where $a_{\kappa}$ is given in 2.8 . 
Proof. We remark that the vector field $D_{t}$ is tangent to the hypersurface $\Gamma$. This means that $D_{t} \tilde{\theta}$ and $D_{t} \tilde{\kappa}$ are intrinsic quantities only depending respectively on the values of $\theta$ and $\kappa$ on $\Gamma$. For this reason, it is possible to compute these quantities, considering a particular parametrization of $\Gamma$.

We consider $\left(t_{0}, y_{0}\right) \in \Gamma$. Up to translation and rotation of the coordinates, we can assume that $t_{0}=0=y_{0}$ and consider a local representation of $\Gamma$ as

$$
z=u(t, x) \text { for } y=(x, z) \text { with } \quad u_{x}(0,0)=0
$$

where $u_{x}$ denotes $\partial u / \partial x$ and the normal at $(0,0)$ is $(0,1)$ in the $(x, y)$ coordinates. We set $u_{x x}=\partial^{2} u / \partial x^{2}, u_{x t}=\partial^{2} u / \partial x \partial t$, and $u_{x x t}=\partial^{3} u / \partial x^{2} \partial t$. In these coordinates, we have

$$
\theta(t, x, u)=\arctan u_{x}, \quad \kappa(t, x, u)=\frac{u_{x x}}{\left(1+u_{x}^{2}\right)^{3 / 2}}, \quad n(t, x, u)=\frac{1}{\sqrt{1+u_{x}^{2}}}\left(-u_{x}, 1\right) \in \mathbb{R}^{2} .
$$

We recall that locally in a neighbourhood of $(0,0)$, the function $u$ satisfies

$$
u_{t}=c\left(t, x, u, \arctan u_{x}\right) \sqrt{1+u_{x}^{2}} .
$$

For $t$ in a neighbourhood of zero, let us define the curve $\gamma$ contained in $\Gamma$ by

$$
\gamma(t)=(t, 0, u(t, 0))
$$

for which we have

$$
\frac{\mathrm{d} \gamma}{\mathrm{d} t}(0)=\left(1,0, u_{t}(0,0)\right)=(1,0, c(0,0,0))
$$

which is exactly the vector field $D_{t}$ evaluated at the origin, because we assume that $u_{x}$ vanishes at the origin. Therefore

$$
D_{t} \tilde{\theta}(0,0,0)=\frac{\mathrm{d}}{\mathrm{d} t}(\tilde{\theta} \circ \gamma)(0)=\frac{\mathrm{d}}{\mathrm{d} t}\left(\arctan u_{x}(t, 0)\right)_{\mid t=0}=u_{x t}(0,0) .
$$

Similarly

$$
D_{t} \tilde{\kappa}(0,0,0)=\frac{\mathrm{d}}{\mathrm{d} t}(\tilde{\kappa} \circ \gamma)(0)=\frac{\mathrm{d}}{\mathrm{d} t}\left(\frac{u_{x x}(t, 0)}{\left(1+u_{x}^{2}(t, 0)\right)^{3 / 2}}\right)_{\mid t=0}=u_{x x t}(0,0) .
$$

Differentiating 3.22) with respect to $x$, we get

$$
u_{x t}=\left(1, u_{x}\right) \cdot \partial_{y} c \sqrt{1+u_{x}^{2}}+\partial_{\theta} c \frac{u_{x x}}{\sqrt{1+u_{x}^{2}}}+c \frac{u_{x x} u_{x}}{\sqrt{1+u_{x}^{2}}},
$$

which implies that at the origin we have

$$
D_{t} \tilde{\theta}(0,0,0)=u_{x t}(0,0)=\tau \cdot \partial_{y} c+\kappa \partial_{\theta} c=D_{\tau} c .
$$

Differentiating now 3.23 with respect to $x$, we get at the origin

$$
\begin{aligned}
u_{x x t}(0,0)= & \left(0, u_{x x}\right) \cdot \partial_{y} c+(1,0) \otimes(1,0): \partial_{y y}^{2} c+2(1,0) \cdot \partial_{y \theta}^{2} c u_{x x}+\partial_{\theta \theta}^{2} c u_{x x}^{2} \\
& +\partial_{\theta} c u_{x x x}+c u_{x x}^{2} \\
= & \kappa n \cdot \partial_{y} c+\tau \otimes \tau: \partial_{y y}^{2} c+2 \kappa \tau \cdot \partial_{y \theta}^{2} c+\kappa^{2} \partial_{\theta \theta}^{2} c+c \kappa^{2}+\partial_{\theta} c u_{x x x} \\
= & a_{\kappa}+\partial_{\theta} c \frac{\partial}{\partial x}\left(\frac{u_{x x}}{\left(1+u_{x}^{2}\right)^{3 / 2}}\right)
\end{aligned}
$$


with $a_{\kappa}$ given in 2.8 . Hence

$$
D_{t} \tilde{\kappa}(0,0,0)=u_{x x t}(0,0)=a_{\kappa}+\left(\partial_{\theta} c\right)\left(\tau \cdot \partial_{y} \tilde{\kappa}\right) .
$$

This ends the proof of the lemma.

LEMMA 3.4 With the notation of Lemma 3.2, we have

$$
-\int_{\Gamma_{t}}\left(\partial_{\theta} c\right)\left(\tau \cdot \partial_{y} \tilde{\kappa}\right) \partial_{\kappa} \varphi=\int_{\Gamma_{t}} D_{\tau}\left(\varphi \partial_{\theta} c\right) \quad \text { with } \quad D_{\tau}=\tau \cdot \partial_{y}+\kappa \partial_{\theta}
$$

where the quantities in the integrands are evaluated at $(t, y), \theta(t, y), \kappa(t, y)$.

Proof. We consider a parametrization $\gamma$ of the connected curve $\Gamma_{t}$ by the curvilinear abscissa $s$; the point $\gamma(s)$ is associated to $s \in \mathbb{R} /(L \mathbb{Z})$ with $L$ the length of $\Gamma_{t}$ and $\mathrm{d} \gamma / \mathrm{d} s=\tau$. Then we have $\tau \cdot \partial_{y} \tilde{\kappa}=(\mathrm{d} \kappa / \mathrm{d} s)(s)$. With an obvious abuse of notation, we denote by $\kappa(s), \theta(s)$ respectively the curvature and angle associated to $s$, i.e. $\kappa(s)=\kappa(t, \gamma(s)), \theta(s)=\theta(t, \gamma(s))$. For a general $C^{1}$ function $G(y, \theta, \kappa)$, we have

$$
\frac{\mathrm{d}}{\mathrm{d} s}(G(\gamma(s), \theta(s), \kappa(s)))=\left\{\tau \cdot \partial_{y}+\kappa \partial_{\theta}+\frac{\mathrm{d} \kappa}{\mathrm{d} s} \partial_{\kappa}\right\} G
$$

where in particular we have used the fact that $\mathrm{d} \theta / \mathrm{d} s=\kappa($ i.e. $\mathrm{d} \tau / \mathrm{d} s=\kappa n)$. We deduce that

$$
\begin{aligned}
\int_{\Gamma_{t}}\left(\partial_{\theta} c\right) \frac{\mathrm{d} \kappa}{\mathrm{d} s} \partial_{\kappa} \varphi & =\int_{\mathbb{R} /(L \mathbb{Z})} \mathrm{d} s\left(\partial_{\theta} c\right)\left\{\frac{\mathrm{d} \kappa}{\mathrm{d} s} \partial_{\kappa} \varphi+\kappa \partial_{\theta} \varphi+\tau \cdot \partial_{y} \varphi-D_{\tau} \varphi\right\} \\
& =\int_{\mathbb{R} /(L \mathbb{Z})} \mathrm{d} s\left(\partial_{\theta} c\right) \frac{\mathrm{d} \varphi}{\mathrm{d} s}-\int_{\mathbb{R} /(L \mathbb{Z})} \mathrm{d} s\left(\partial_{\theta} c\right) D_{\tau} \varphi \\
& =-\int_{\mathbb{R} /(L \mathbb{Z})} \mathrm{d} s\left\{\left(\frac{\mathrm{d}}{\mathrm{d} s}\left(\partial_{\theta} c\right)\right) \varphi+\left(\partial_{\theta} c\right) D_{\tau} \varphi\right\} \\
& =-\int_{\mathbb{R} /(L \mathbb{Z})} \mathrm{d} s\left\{\left(D_{\tau}\left(\partial_{\theta} c\right)\right) \varphi+\left(\partial_{\theta} c\right) D_{\tau} \varphi\right\}=-\int_{\Gamma_{t}} D_{\tau}\left(\varphi \partial_{\theta} c\right)
\end{aligned}
$$

where we have used (3.24) with $G=\varphi$ in the second line, we have made an integration by parts in the third line, and used (3.24) with $G=\partial_{\theta} c$ in the fourth line. This ends the proof of the lemma.

LEMMA 3.5 For a general solution $g$ of 2.6, we have

$$
\left(D_{\tau} g\right)_{t}+\partial_{y} \cdot\left(a_{y} D_{\tau} g\right)+\partial_{\theta}\left(a_{\theta} D_{\tau} g\right)+\partial_{\kappa}\left(D_{\tau}\left(a_{\kappa} g\right)\right)=0
$$

Proof. For two vector fields $V_{1}, V_{2}$ and a given distribution $g$, we recall the definition of brackets $\left[V_{1}, V_{2}\right]$ :

$$
\left[V_{1}, V_{2}\right] g=V_{1}\left(V_{2} g\right)-V_{2}\left(V_{1} g\right) .
$$

We first compute the following brackets of vector fields for general $g$ :

$$
\left\{\begin{array}{l}
{\left[D_{\tau}, \partial_{y} \cdot\left(a_{y} \cdot\right)\right] g=\left(D_{\tau}\left(\partial_{y} \cdot a_{y}\right)\right) g+\left(D_{\tau} a_{y}\right) \cdot \partial_{y} g+a_{y} \cdot\left[D_{\tau}, \partial_{y}\right] g} \\
{\left[D_{\tau}, \partial_{\theta}\left(a_{\theta} \cdot\right)\right] g=\left(D_{\tau}\left(\partial_{\theta} a_{\theta}\right)\right) g+\left(D_{\tau} a_{\theta}\right) \partial_{\theta} g+a_{\theta}\left[D_{\tau}, \partial_{\theta}\right] g}
\end{array}\right.
$$


and

$$
\left\{\begin{array}{l}
{\left[D_{\tau}, \partial_{y}\right]=0} \\
{\left[D_{\tau}, \partial_{\theta}\right]=-n \cdot \partial_{y}} \\
{\left[D_{\tau}, \partial_{\kappa}\right]=-\partial_{\theta}}
\end{array}\right.
$$

Applying the vector field $D_{\tau}$ to the equation

$$
-g_{t}=a_{0} g+\partial_{y} \cdot\left(a_{y} g\right)+\partial_{\theta}\left(a_{\theta} g\right)+\partial_{\kappa}\left(a_{\kappa} g\right)
$$

we get

$$
\begin{aligned}
-\left(D_{\tau} g\right)_{t}= & D_{\tau}\left(a_{0} g\right)+\partial_{y} \cdot\left(a_{y} D_{\tau} g\right)+\partial_{\theta}\left(a_{\theta} D_{\tau} g\right)+\partial_{\kappa}\left(D_{\tau}\left(a_{\kappa} g\right)\right) \\
& +\left[D_{\tau}, \partial_{y} \cdot\left(a_{y} \cdot\right)\right] g+\left[D_{\tau}, \partial_{\theta}\left(a_{\theta} \cdot\right)\right] g+\left[D_{\tau}, \partial_{\kappa}\right]\left(a_{\kappa} g\right),
\end{aligned}
$$

i.e.

$$
\begin{aligned}
&-\left(D_{\tau} g\right)_{t}-\partial_{y} \cdot\left(a_{y} D_{\tau} g\right)-\partial_{\theta}\left(a_{\theta} D_{\tau} g\right)-\partial_{\kappa}\left(D_{\tau}\left(a_{\kappa} g\right)\right) \\
&= D_{\tau}\left(a_{0} g\right)+\left[D_{\tau}, \partial_{y} \cdot\left(a_{y} \cdot\right)\right] g+\left[D_{\tau}, \partial_{\theta}\left(a_{\theta} \cdot\right)\right] g+\left[D_{\tau}, \partial_{\kappa}\right]\left(a_{\kappa} g\right) \\
&=\left(D_{\tau} a_{0}\right) g+a_{0} D_{\tau} g+\left(D_{\tau}\left(\partial_{y} \cdot a_{y}\right)\right) g+\left(D_{\tau} a_{y}\right) \cdot \partial_{y} g \\
&+\left(D_{\tau}\left(\partial_{\theta} a_{\theta}\right)\right) g+\left(D_{\tau} a_{\theta}\right) \partial_{\theta} g-a_{n} \cdot \partial_{y} g-\partial_{\theta}\left(a_{\kappa} g\right) \\
&=\left(D_{\tau} a_{0}+D_{\tau}\left(\partial_{y} \cdot a_{y}\right)+D_{\tau}\left(\partial_{\theta} a_{\theta}\right)-\partial_{\theta} a_{\kappa}\right) g \\
&+\left(a_{0} \tau+D_{\tau} a_{y}-a_{n}\right) \cdot \partial_{y} g+\left(a_{0} \kappa+D_{\tau} a_{\theta}-a_{\kappa}\right) \partial_{\theta} g \\
&= 0
\end{aligned}
$$

because, on the other hand, we compute

$$
\left\{\begin{array}{l}
D_{\tau} a_{\theta}=a_{\kappa}-a_{0} \kappa \\
D_{\tau} a_{y}=a_{n}-a_{0} \tau \\
D_{\tau} a_{0}+D_{\tau}\left(\partial_{y} \cdot a_{y}\right)+D_{\tau}\left(\partial_{\theta} a_{\theta}\right)-\partial_{\theta} a_{\kappa}=0
\end{array}\right.
$$

where the last line is a consequence of the following computations:

$$
\left\{\begin{array}{l}
\partial_{y} \cdot a_{y}+\partial_{\theta} a_{\theta}=2 n \cdot \partial_{y} c \\
D_{\tau}\left(\partial_{y} \cdot a_{y}+\partial_{\theta} a_{\theta}\right)=-2\left(\kappa \tau \cdot \partial_{y} c-\kappa n \cdot \partial_{y \theta}^{2} c-\partial_{y y}^{2} c \cdot(\tau, n)\right) \\
D_{\tau} a_{0}-\partial_{\theta} a_{\kappa}=\tau \cdot \partial_{y} a_{0}-\partial_{\theta}\left(D_{\tau} a_{\theta}\right)=2\left(\kappa \tau \cdot \partial_{y} c-\kappa n \cdot \partial_{y \theta}^{2} c-\partial_{y y}^{2} c \cdot(\tau, n)\right) .
\end{array}\right.
$$

This ends the proof of the lemma.

Proof of Theorem 2.1. Putting together the results of Lemmata 3.2, 3.3 and 3.4. we get, for $g=g_{\Gamma}$,

$$
\begin{aligned}
\left\langle g_{t}, \varphi\right\rangle & =\left\langle g,-c \kappa \varphi+c n \cdot \partial_{y} \varphi+\left(D_{\tau} c\right) \partial_{\theta} \varphi+a_{\kappa} \partial_{\kappa} \varphi+\left(\partial_{\theta} c\right)\left(\tau \cdot \partial_{y} \tilde{\kappa}\right) \partial_{\kappa} \varphi\right\rangle \\
& =\left\langle g,-c \kappa \varphi+c n \cdot \partial_{y} \varphi+\left(D_{\tau} c\right) \partial_{\theta} \varphi+a_{\kappa} \partial_{\kappa} \varphi-\left(\partial_{\theta} c\right) D_{\tau} \varphi-\varphi D_{\tau}\left(\partial_{\theta} c\right)\right\rangle \\
& =\left\langle g,-a_{0} \varphi+a_{y} \partial_{y} \varphi+a_{\theta} \partial_{\theta} \varphi+a_{\kappa} \partial_{\kappa} \varphi\right\rangle,
\end{aligned}
$$


which leads to equation (2.6). Integrating equation (3.25) with respect to $\kappa$ leads to (2.9). Finally, for $g_{\Gamma}$ defined in (2.4) and $\psi(t, y, \theta, \kappa)=\psi(t, y, \theta)$ we compute

$$
\begin{aligned}
\left\langle\hat{g}_{\Gamma}, \psi\right\rangle & =\left\langle D_{\tau} g, \bar{\psi}\right\rangle=-\left\langle g_{\Gamma}, D_{\tau} \bar{\psi}\right\rangle=-\left\langle g_{\Gamma}, \tau \cdot \partial_{y} \bar{\psi}+\kappa \partial_{\theta} \bar{\psi}+\frac{\mathrm{d} \kappa}{\mathrm{d} s} \partial_{\kappa} \bar{\psi}\right\rangle \\
& =-\int_{0}^{T} \mathrm{~d} t \int_{\Gamma_{t}} \tau \cdot \partial_{y} \tilde{\psi}(t, y)=0
\end{aligned}
$$

where in the third equality we have used the fact that $\bar{\psi}$ is independent of $\kappa$, and in the fourth equality we have used 3.24 with $G=\bar{\psi}$. Finally, we have set $\tilde{\psi}(t, y)=\bar{\psi}(t, y, \theta(t, y), \kappa(t, y))$. This proves that $\hat{g}_{\Gamma}=0$. This ends the proof of the theorem.

Proof of Propositon 2.2. We first multiply by $\tau$ the equation (2.6) satisfied by $g$ to get

$$
0=(\tau g)_{t}+a_{0} \tau g+\partial_{y} \cdot\left(a_{y} \otimes \tau g\right)+\partial_{\theta}\left(a_{\theta} \tau g\right)-a_{\theta} n g+\partial_{\kappa}\left(a_{\kappa} \tau g\right) .
$$

We compute

$$
\begin{aligned}
& a_{0} \tau g+ \partial_{y} \cdot\left(a_{y} \otimes \tau g\right)-a_{\theta} n g+\partial_{\theta}(c \kappa n g) \\
&=\partial_{y} \cdot\left(a_{y} \otimes \tau g\right)-a_{\theta} n g+n \partial_{\theta}(c \kappa g)+\kappa\left(\partial_{\theta \theta}^{2} c\right) \tau g+\left(\tau \cdot \partial_{y \theta}^{2} c\right) \tau g \\
&=\left(n \cdot \partial_{y}\right)(c \tau g)-\left(\tau \cdot \partial_{y}\right)\left(\left(\partial_{\theta} c\right) \tau g\right)-\left(\tau \cdot \partial_{y} c\right) n g \\
&+n c \kappa \partial_{\theta} g+n \kappa\left(\partial_{\theta} c\right) g+\left\{\partial_{\theta}\left(\kappa\left(\partial_{\theta} c\right) \tau g\right)-\kappa\left(\partial_{\theta} c\right) n g-\kappa\left(\partial_{\theta} c\right) \tau \partial_{\theta} g\right\}+\left(\tau \cdot \partial_{y \theta}^{2} c\right) \tau g \\
&= n c D_{\tau} g-n c \tau \cdot \partial_{y} g-\left(\tau \cdot \partial_{y} c\right) n g+\left(n \cdot \partial_{y}\right)(c \tau g)-\left(\tau \cdot \partial_{y}\right)\left(\left(\partial_{\theta} c\right) \tau g\right) \\
&+\partial_{\theta}\left(\kappa\left(\partial_{\theta} c\right) \tau g\right)-\left(\partial_{\theta} c\right) \tau D_{\tau} g+\left(\partial_{\theta} c\right) \tau\left(\tau \cdot \partial_{y} g\right)+\left(\tau \cdot \partial_{y \theta}^{2} c\right) \tau g \\
&= \partial_{\theta}\left(\kappa\left(\partial_{\theta} c\right) \tau g\right)+\left(D_{\tau} g\right)\left(c n-\tau \partial_{\theta} c\right)-n\left(\tau \cdot \partial_{y}\right)(c g)+\tau\left(n \cdot \partial_{y}\right)(c g) \\
&= \partial_{\theta}\left(\kappa\left(\partial_{\theta} c\right) \tau g\right)+\left(D_{\tau} g\right)\left(c n-\tau \partial_{\theta} c\right)-\partial_{y}^{\perp}(c g)
\end{aligned}
$$

where we have used the explicit expression of $a_{0}$ in the second line, the explicit expressions of $a_{y}$ and $a_{\theta}$ in the third line, and we have introduced the expression of $D_{\tau} g=\tau \cdot \partial_{y} g+\kappa \partial_{\theta} g$ in the fourth line. Therefore we get

$$
0=(\tau g)_{t}-\partial_{y}^{\perp}(c g)+\left(D_{\tau} g\right) a_{y}+\partial_{\theta}\left(\left(a_{\theta}+\kappa \partial_{\theta} c\right) \tau g-c \kappa n g\right)+\partial_{\kappa}\left(a_{\kappa} \tau g\right) .
$$

This ends the proof of the proposition.

\section{Result in dimension $N \geqslant 2$}

We now return to our main result in dimension $N \geqslant 2$. We use the notation of Subsection 1.3 We consider a closed connected and oriented hypersurface $\Gamma_{t}$ for $t \in[0, T)$ for some fixed $T>0$, with the normal pointing outwards from the bounded set whose boundary is the hypersurface. At a point $y$ of $\Gamma_{t}, n(t, y)$ is the unit normal and $K(t, y) \in \mathbb{R}_{\mathrm{sym}}^{N \times N}$ the curvature matrix (negative for a ball).

For $(t, y, n, K) \in[0, T) \times \mathbb{R}^{N} \times \mathbb{S}^{N-1} \times \mathbb{R}_{\mathrm{sym}}^{N \times N}$, we define the measure $g_{\Gamma}(t, y, n, K)$ by

$$
\left\langle g_{\Gamma}, \varphi\right\rangle=\int_{0}^{T} \mathrm{~d} t \int_{\Gamma_{t}} \varphi(t, y, n(t, y), K(t, y))
$$


for any test function $\varphi \in C_{c}^{\infty}\left([0, T) \times \mathbb{R}^{N} \times \mathbb{S}^{N-1} \times \mathbb{R}_{\mathrm{sym}}^{N \times N}\right)$. Given any distribution $g$ (with compact support in the variable $K \in \mathbb{R}_{\text {sym }}^{N \times N}$ ), we also define formally the distribution $\hat{g}(t, y, n)$ by

$$
\hat{g}:=\int_{\mathbb{R}_{\text {sym }}^{N \times N}} \mathrm{~d} K\left\{D_{\tau} g+n(I: K) g\right\} \quad \text { with } \quad D_{\tau}=-(I-n \otimes n) \cdot \partial_{y}+K \cdot \partial_{n},
$$

i.e. rigorously, for any $\psi \in C_{c}^{\infty}\left([0, T) \times \mathbb{R}^{N} \times \mathbb{S}^{N-1}\right)$,

$$
\langle\hat{g}, \psi\rangle=\left\langle D_{\tau} g+n(I: K) g, \bar{\psi}\right\rangle \quad \text { with } \quad \bar{\psi}(t, y, n, K)=\psi(t, y, n) .
$$

Then we have

THEOREM 4.1 (Equivalence geometric motion/linear transport, $N \geqslant 2$ ) Under the regularity assumption (1.2), if $\left(\Gamma_{t}\right)_{t}$ solves equation (1.1) on the time interval $[0, T)$, then the distribution $g_{\Gamma}(t, y, n, K)$ defined by 4.26 , solves the equation

$$
g_{t}+\operatorname{div}(a g)+a_{0} g=0 \quad \text { in } \quad \mathcal{D}^{\prime}\left((0, T) \times \mathbb{R}^{N} \times \mathbb{S}^{N-1} \times \mathbb{R}_{\mathrm{sym}}^{N \times N}\right)
$$

with

$$
\operatorname{div}(a g)=\partial_{y} \cdot\left(a_{y} g\right)+\partial_{n} \cdot\left(a_{n} g\right)+\partial_{K}:\left(a_{K} g\right) \quad \text { for } a=\left(a_{y}, a_{n}, a_{K}\right)
$$

and

$$
\left\{\begin{aligned}
a_{0}= & c(I: K)+K: \partial_{n n}^{2} c-I: \partial_{n y}^{2} c, \quad a_{y}=c n+\partial_{n} c, \quad a_{n}=-(I-n \otimes n) \cdot \partial_{y} c, \\
a_{K}= & c K^{2}+K \cdot \partial_{n n}^{2} c \cdot K+(I-n \otimes n) \cdot \partial_{y y}^{2} c \cdot(I-n \otimes n) \\
& +\left(n \cdot \partial_{y} c\right) K-K \cdot \partial_{n y}^{2} c \cdot(I-n \otimes n)-(I-n \otimes n) \cdot \partial_{y n}^{2} c \cdot K \\
& +K \cdot \partial_{y} c \otimes n+n \otimes K \cdot \partial_{y} c .
\end{aligned}\right.
$$

Moreover, for $\alpha:=K \cdot n g$ we have

$$
\alpha_{t}+\operatorname{div}(a \otimes \alpha)+A_{0} \cdot \alpha=0 \quad \text { in }\left(\mathcal{D}^{\prime}\left((0, T) \times \mathbb{R}^{N} \times \mathbb{S}^{N-1} \times \mathbb{R}_{\mathrm{sym}}^{N \times N}\right)\right)^{N}
$$

with

$$
-A_{0}=c K+K \cdot \partial_{n n} c-(I-n \otimes n) \cdot \partial_{y n}^{2} c+n \otimes \partial_{y} c+\left(2\left(n \cdot \partial_{y} c\right)-a_{0}\right) I .
$$

Finally, for $g_{\Gamma}$ defined by (4.26) we have $K \cdot n g_{\Gamma}=0$ and $\hat{g}_{\Gamma}$ defined in 4.27) satisfies $\hat{g}_{\Gamma}=0$.

Let us now make a few comments on this theorem.

The invariant manifold. We first remark that in the expression (4.30) of $a_{K}$, the last two terms are new in comparison to 22.87 . Moreover, these terms are the only terms not perpendicular to $n$. Their existence is due to our choice of writing the equations on $\mathbb{R}^{N} \times \mathbb{S}^{N-1} \times \mathbb{R}_{\mathrm{sym}}^{N \times N}$, in order to preserve $K \cdot n=0$ on the support of $g$ for all time, if it is true at the initial time (see the justification of equation (4.31)). This really means that we are interested in measures with support on the natural manifold

$$
\mathcal{M}=\left\{(y, n, K) \in \mathbb{R}^{N} \times \mathbb{S}^{N-1} \times \mathbb{R}_{\mathrm{sym}}^{N \times N}: K \cdot n=0\right\},
$$

whose dimension is the same as that of $\mathbb{R}^{N} \times \mathbb{S}^{N-1} \times \mathbb{R}_{\mathrm{sym}}^{(N-1) \times(N-1)}$. This is obviously related to 
the fact (easy to check) that for any $t \in[0, T)$, the vector field

$$
a^{t}(X)=a(t, X) \quad \text { with } \quad X=(y, n, K)
$$

is tangent to the manifold $\mathcal{M}$. This means that it should be possible to represent (but probably less simple to write) the transport equation as some transport equation on the manifold $\mathcal{M}$, similar to equation 4.28). This also means that, while we keep our description on the space $\mathbb{R}^{N} \times \mathbb{S}^{N-1} \times$ $\mathbb{R}_{\mathrm{sym}}^{N \times N}$, there are several equivalent transport formulations, because for what we have in mind, in 4.30), $K$ can be replaced by $K \cdot(I-n \otimes n)$, or $(I-n \otimes n) \cdot K$, or even $(I-n \otimes n) \cdot K \cdot(I-n \otimes n)$.

Explicit solution based on characteristics. It is known (see for instance Lions [22] or LeVeque [21]) that the solution $g$ of 4.28 is given (at least formally with the measure $g(0, \cdot)$ at the initial time) for $X=(y, n, K)$ by

$$
g(t, Y)=g(0, X(0 ; t, Y)) e^{-\int_{0}^{t} b_{0}(s ; t, Y) \mathrm{d} s} \quad \text { with } \quad b_{0}(s ; t, Y)=\left(a_{0}+\operatorname{div} a\right)(s, X(s ; t, Y))
$$

and

$$
\frac{\mathrm{d}}{\mathrm{d} s} X(s ; t, Y)=a(s, X(s ; t, Y)), \quad X(t ; t, Y)=Y .
$$

In particular, defining for $t \geqslant 0$,

$$
\hat{\Gamma}_{t}=\left\{\begin{array}{l}
(y, n(t, y), K(t, y)) \in \mathbb{R}^{N} \times \mathbb{S}^{N-1} \times \mathbb{R}_{\mathrm{sym}}^{N \times N} \\
\text { for } y \in \Gamma_{t} \text { with normal } n(t, y) \text { and curvature } K(t, y)
\end{array}\right\}
$$

we have

$$
\operatorname{supp} g_{\Gamma}(t, \cdot)=\hat{\Gamma}_{t} .
$$

Therefore, if $Y \in \hat{\Gamma}_{0}$, then $X(t ; 0, Y) \in \hat{\Gamma}_{t}$, which shows (at least formally) that the solutions $X(t ; 0, Y)$ are the characteristics of the evolution. In particular, we get, as a result, the value of the evolution of the curvature, which has to be related to curvature estimates in Cannarsa and Frankowska [5], Cannarsa and Cardaliaguet [4] or Alvarez, Cardaliaguet and Monneau [1]. Moreover, at least the first two components $\left(c n+\partial_{n} c,-(I-n \otimes n) \cdot \partial_{y} c\right)$ of this vector field are similar to the characteristics of classical Hamilton-Jacobi equations with Hamiltonian $c(t, y, n)$ (see Evans [9]).

The mathematical difficulty for long time existence. The principal mathematical difficulty in solving equation (4.28) is the quadratic growth of the vector field $a$ as a function of the curvature $K$. This means that, even for initial data with compact support, the support of the solution can go to infinity in $K$ in finite time (which corresponds to the appearance of geometric singularities of fronts, revealed by infinite curvature). On the contrary, in the particular case where the hypersurface is transported by a vector field $V(t, y)$, which means that the normal velocity is given by $c(t, y, n)=n \cdot V(t, y)$, we can check that the vector field $a$ is at most linear in the curvature $K$, and $a_{y}=V$ is exactly the original vector field. This is natural, because it is well-known that linear transport equations do not create singularities in finite time. See also the appendix (Subsection 6.3 and Remark 6.5 for indications how to overcome these difficulties using a different transport formulation.

We do not know if there are conservative quantities (other than $K \cdot n g$, like maybe $\hat{g}$ ) that can be derived from this transport formulation of moving fronts. We do not know either what is the best regularity that we can assume on $g$, in order to satisfy the natural constraints like the conserved quantities. 
5. Proofs in dimension $N \geqslant 2$

We start with the following result which, for completeness, is proved in the appendix (Section 6).

LEMMA 5.1 Let $\psi \in C_{c}^{1}\left((0, T) \times \mathbb{R}^{N}\right)$ and $\left(\Gamma_{t}\right)_{t}$ be a smooth evolution with normal velocity $c(t, y)$. Then

$$
\frac{\mathrm{d}}{\mathrm{d} t}\left(\int_{\Gamma_{t}} \psi\right)=\int_{\Gamma_{t}} D_{t} \psi-c(I: K) \psi \quad \text { with } \quad D_{t} \psi:=\psi_{t}+c n \cdot \partial_{y} \psi
$$

where $\psi, n, K$ and $c$ are evaluated at the current point $(t, y)$, and $I: K$ denotes the trace of the matrix $K$.

Then we have the following result:

LEMMA 5.2 For any $\varphi \in C_{c}^{\infty}\left((0, T) \times \mathbb{R}^{N} \times \mathbb{S}^{N-1} \times \mathbb{R}_{\mathrm{sym}}^{N \times N}\right)$ and $g=g_{\Gamma}$, we have

$$
\left\langle g_{t}, \varphi\right\rangle=\left\langle g,-c(I: K) \varphi+c n \cdot \partial_{y} \varphi+\left(D_{t} \tilde{n}\right) \cdot \partial_{n} \varphi+\left(D_{t} \tilde{K}\right): \partial_{K} \varphi\right\rangle
$$

for any $C^{1}$ extension $\tilde{n}$ (resp. $\left.\tilde{K}\right)$ of $n$ (resp. $K$ ).

Proof. The proof is similar to the proof of Lemma 3.2. with 3.20) replaced by 5.33).

LEMMA 5.3 Consider a local parametrization of the hypersurface $\Gamma$ as

$$
z=u(t, x) \quad \text { for } y=(x, z) \in U \subset \mathbb{R}^{N-1} \times \mathbb{R}
$$

where $U$ is an open set. Then with $u_{x}=\partial u / \partial x \in \mathbb{R}^{N-1}$ and $u_{x x}=\partial^{2} u / \partial x^{2} \in \mathbb{R}_{\text {sym }}^{(N-1) \times(N-1)}$ we have

$$
n(t, x, u)=\frac{1}{\sqrt{1+u_{x}^{2}}}\left(-u_{x}, 1\right) \in \mathbb{R}^{N-1} \times \mathbb{R} \quad \text { and } \quad K(t, x, u)=F\left(u_{x x}, u_{x}\right) \in \mathbb{R}_{\mathrm{sym}}^{N \times N}
$$

where for any $M \in \mathbb{R}_{\mathrm{sym}}^{(N-1) \times(N-1)}$ and $p \in \mathbb{R}^{N-1}$,

$$
\begin{aligned}
F(M, p)=\frac{1}{\sqrt{1+p^{2}}}\left\{\mathcal{I}(M)+\frac{p \cdot M \cdot p}{\left(1+p^{2}\right)^{2}}(-p, 1) \otimes(-p, 1)\right. \\
\left.\quad+\left(\frac{M \cdot p}{1+p^{2}}, 0\right) \otimes(-p, 1)+(-p, 1) \otimes\left(\frac{M \cdot p}{1+p^{2}}, 0\right)\right\}
\end{aligned}
$$

with

$$
(\mathcal{I}(M))_{i j}= \begin{cases}M_{i j} & \text { if } i, j \in\{1, \ldots, n-1\} \\ 0 & \text { if } i=n \text { or } j=n\end{cases}
$$

Proof. The only thing to prove is the expression of the curvature. In the case where $n=(0,1) \in$ $\mathbb{R}^{N-1} \times \mathbb{R}$, i.e. $u_{x}=0$, it is clear that $F(M, 0)=\mathcal{I}(M)$. In the general case where $n \neq(0,1)$, i.e. $u_{x} \neq 0$, we need to make a rotation of the coordinates in the plane generated by $n$ and $(0,1)$, such that in the new coordinates the surface $\Gamma$ is represented by $Z=v(t, X)$ with $v_{X}=0$. Then a full computation (based on the inverse function theorem) is possible. 
We now give the details of the computation. We drop the time coordinate which does not play any role in this computation. Up to a translation we can assume that we work close to the origin where $u_{x}(0) \neq 0$. We choose an orthonormal basis $\left(e_{1}, \ldots, e_{N-1}\right)$ such that

$$
e_{1}=\frac{u_{x}}{\left|u_{x}\right|}(0) \text {. }
$$

We set

$$
x^{\prime}=\left(x_{2}, \ldots, x_{N-1}\right)=X^{\prime} .
$$

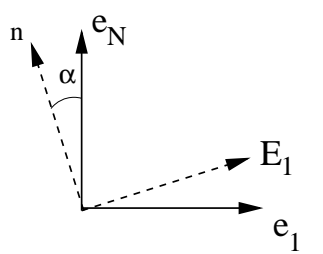

FIG. 1. New coordinates by rotation.

We will consider the unit vector $E_{1}$ such that the orthonormal basis $\left(E_{1}, n(0)\right)$ of the plane is obtained by a rotation of the basis $\left(e_{1}, e_{N}\right)$ (see Fig. 1), and define the new coordinates $X_{1}, X_{N}$ such that

$$
X_{1} E_{1}+X_{N} n(0)=x_{1} e_{1}+x_{N} e_{N} \text { and } X=\left(X_{1}, X^{\prime}, X_{N}\right),
$$

i.e.

$$
X_{1}=x_{1} \cos \alpha+x_{N} \sin \alpha, \quad X_{N}=-x_{1} \sin \alpha+x_{N} \cos \alpha
$$

for

$$
\alpha=\operatorname{angle}\left(e_{N}, n(0)\right) \in(0, \pi / 2), \quad \text { i.e. } \quad \cos \alpha=\frac{1}{\sqrt{1+u_{1}^{2}}}, \quad \sin \alpha=\frac{u_{1}}{\sqrt{1+u_{1}^{2}}}
$$

with $u_{1}=\partial u / \partial x_{1}$. We see that

$$
x_{N}=u\left(x_{1}, x^{\prime}\right) \Leftrightarrow X_{N}=v\left(X_{1}, X^{\prime}\right)
$$

for some new function $v$ which satisfies

$$
v\left(x_{1} \cos \alpha+u\left(x_{1}, x^{\prime}\right) \sin \alpha, x^{\prime}\right)=-x_{1} \sin \alpha+u\left(x_{1}, x^{\prime}\right) \cos \alpha .
$$

We compute by differentiation

$$
\left\{\begin{array}{l}
v_{1}\left(\cos \alpha+u_{1} \sin \alpha\right)=-\sin \alpha+u_{1} \cos \alpha \\
v_{1} u_{i} \sin \alpha+v_{i}=u_{i} \cos \alpha, \quad i=2, \ldots, N-1
\end{array}\right.
$$

In particular we have $v_{X}(0)=0$. Differentiating once again, we get at the origin

$$
\left\{\begin{aligned}
v_{11} & =\frac{u_{11}}{\left(1+u_{1}^{2}\right)^{3 / 2}}, \\
v_{1 i} & =\frac{u_{1 i}}{1+u_{1}^{2}}, \quad i=2, \ldots, N-1, \\
v_{i j} & =\frac{u_{i j}}{\left(1+u_{1}^{2}\right)^{1 / 2}}, \quad i, j=2, \ldots, N-1 .
\end{aligned}\right.
$$


Then $\left(\right.$ with $\left.E_{1}=(\cos \alpha) e_{1}+(\sin \alpha) e_{N}, E_{1}^{\prime}=(\cos \alpha) E_{1}\right)$

$$
\begin{aligned}
K\left(1+u_{1}^{2}\right)^{1 / 2}= & v_{11} E_{1} \otimes E_{1}+\sum_{i=2}^{N-1} v_{1 i}\left(E_{1} \otimes e_{i}+e_{i} \otimes E_{1}\right)+\sum_{i, j=2}^{N-1} v_{i j} e_{i} \otimes e_{j} \\
= & \sum_{i, j=1}^{N-1} u_{i j} e_{i} \otimes e_{j}+\sum_{i=1}^{N-1} u_{1 i}\left(\left(E_{1}^{\prime}-e_{1}\right) \otimes e_{i}+e_{i} \otimes\left(E_{1}^{\prime}-e_{1}\right)\right) \\
& +u_{11}\left(E_{1}^{\prime}-e_{1}\right) \otimes\left(E_{1}^{\prime}-e_{1}\right)
\end{aligned}
$$

Using the fact that

$$
E_{1}^{\prime}-e_{1}=\frac{u_{1}}{1+u_{1}^{2}}\left(-u_{1} e_{1}+e_{N}\right)
$$

we see that we get exactly $K=F\left(u_{x x}, u_{1} e_{1}\right)$, which ends the proof of the lemma.

LEMMA 5.4 With the notation of Lemma 5.2, on $\Gamma$ we have

$$
\left\{\begin{array}{l}
D_{t} \tilde{n}=-(I-n \otimes n) \cdot \partial_{y} c+K \cdot \partial_{n} c=: D_{\tau} c \\
D_{t} \tilde{K}=a_{K}-\partial_{n} c \cdot \partial_{y} \tilde{K}
\end{array}\right.
$$

with $a_{K}$ given in 4.30, and on $\Gamma$,

$$
(I-n \otimes n) \cdot \partial_{y} \tilde{n}=-K .
$$

Proof. We proceed exactly as in the proof of Lemma 3.3 We consider $\left(t_{0}, y_{0}\right) \in \Gamma$. Up to translation and rotation of coordinates, we can assume that $t_{0}=0=y_{0}$ and consider a local representation of $\Gamma$ as

$$
z=u(t, x) \quad \text { for } y=(x, z) \quad \text { with } \quad u_{x}(0,0)=0
$$

where $u_{x}$ denotes $\partial u / \partial x$ and the normal at $(0,0)$ is $(0,1)$ in the $(x, y)$ coordinates. We set $u_{x x}=\partial^{2} u / \partial x^{2}, u_{x t}=\partial^{2} u / \partial x \partial t$, and $u_{x x t}=\partial^{3} u / \partial^{2} x \partial t$. In these coordinates, the angle $n$ and the curvature $K$ are given by Lemma 5.3 .

We recall that locally in a neighbourhood of $(0,0)$, the function $u$ satisfies

$$
u_{t}=c(t, x, u, n) \sqrt{1+u_{x}^{2}} .
$$

For $t$ in a neighbourhood of zero, let us define the curve $\gamma$ contained in $\Gamma$ by

$$
\gamma(t)=(t, 0, u(t, 0)) \text {. }
$$

Then

$$
\begin{aligned}
D_{t} \tilde{n}(0,0,0) & =\frac{\mathrm{d}}{\mathrm{d} t}(\tilde{n} \circ \gamma)(0)=\frac{\mathrm{d}}{\mathrm{d} t}(n(t, 0, u(t, 0)))_{\mid t=0} \\
& =\frac{\mathrm{d}}{\mathrm{d} t}\left(\frac{1}{\sqrt{1+u_{x}^{2}(t, 0)}}\left(-u_{x}(t, 0), 1\right)\right)_{\mid t=0}=\left(-u_{x t}(0,0), 0\right) .
\end{aligned}
$$


Similarly

$$
\begin{aligned}
D_{t} \tilde{K}(0,0,0) & =\frac{\mathrm{d}}{\mathrm{d} t}(\tilde{K} \circ \gamma)(0)=\frac{\mathrm{d}}{\mathrm{d} t}\left(F\left(u_{x x}(t, 0), u_{x}(t, 0)\right)_{\mid t=0}\right. \\
& =F_{M}^{\prime}\left(\cdot, u_{x}(0,0)\right) u_{x x t}(0,0)+F_{p}^{\prime}\left(u_{x x}(0,0), u_{x}(0,0)\right) u_{x t}(0,0) \\
& =\mathcal{I}\left(u_{x x t}\right)+\left(u_{x x} \cdot u_{x t}, 0\right) \otimes(0,1)+(0,1) \otimes\left(u_{x x} \cdot u_{x t}, 0\right)
\end{aligned}
$$

where all the quantities are evaluated at the origin $(0,0)$.

Differentiating 3.22, with respect to $x$, we get

$$
u_{x t}=\left(\partial_{x} c+u_{x} \partial_{y_{N}} c+\partial_{n} c \cdot n_{x}\right) \sqrt{1+u_{x}^{2}}+c \frac{u_{x x} \cdot u_{x}}{\sqrt{1+u_{x}^{2}}}
$$

where

$$
n_{x}=-\frac{1}{\left(1+u_{x}^{2}\right)^{3 / 2}}\left(-u_{x}, 1\right) \otimes u_{x x} \cdot u_{x}+\frac{1}{\sqrt{1+u_{x}^{2}}}\left(-u_{x x}, 0\right),
$$

which implies that at the origin we have

$$
D_{t} \tilde{n}(0,0,0)=\left(-u_{x t}(0,0), 0\right)=-(I-n \otimes n) \cdot \partial_{y} c+K \cdot \partial_{n} c=D_{\tau} c
$$

and

$$
(I-n \otimes n) \cdot \partial_{y} \tilde{n}=-K .
$$

Differentiating now (5.37) with respect to $x$, we get at the origin (with contraction in $n$ in expressions like $n_{x} \cdot \partial_{n x}^{2} c$ or $\left.n_{x} \cdot \partial_{n n}^{2} c \cdot n_{x}\right)$

$$
u_{x x t}(0,0)=\partial_{x x}^{2} c+\partial_{x n}^{2} c \cdot n_{x}+u_{x x} \partial_{y_{N}} c+n_{x} \cdot \partial_{n x}^{2} c+n_{x} \cdot \partial_{n n}^{2} c \cdot n_{x}+\partial_{n} c \cdot n_{x x}+c u_{x x} \cdot u_{x x}
$$

and then

$$
\mathcal{I}\left(u_{x x t}\right)=A+\mathcal{I}\left(\partial_{n} c \cdot n_{x x}\right)
$$

with

$$
\begin{aligned}
A= & (I-n \otimes n) \cdot\left\{\partial_{y y}^{2} c-\partial_{y n}^{2} c \cdot K+K\left(n \cdot \partial_{y} c\right)-K \cdot \partial_{n y}^{2} c+K \cdot \partial_{n n}^{2} c \cdot K+c K \cdot K\right\} \\
& \cdot(I-n \otimes n) .
\end{aligned}
$$

Hence

$$
D_{t} \tilde{K}(0,0,0)=B+C
$$

with

$$
B=A+K \cdot D_{\tau} c \otimes n+n \otimes K \cdot D_{\tau} c+K^{2} \cdot \partial_{n} c \otimes n+n \otimes K^{2} \cdot \partial_{n} c=a_{K}
$$

where $a_{K}$ given in 4.30 and

$$
C=\mathcal{I}\left(\partial_{n} c \cdot n_{x x}\right)-K^{2} \cdot \partial_{n} c \otimes n-n \otimes K^{2} \cdot \partial_{n} c .
$$

On the one hand, at the origin

$$
n_{x x}=-\left\{(0,1) \otimes u_{x x} \cdot u_{x x}+\left(u_{x x x}, 0\right)\right\}
$$


and then (because $\partial_{n} c$ is orthogonal to $n$ )

$$
\partial_{n} c \cdot n_{x x}=-u_{x x x} \cdot \partial_{n} c .
$$

On the other hand, at the origin

$$
\begin{aligned}
\left(F\left(u_{x x}, u_{x}\right)\right)_{x} & =F_{M}^{\prime}\left(\cdot, u_{x}\right) u_{x x x}+F_{p}^{\prime}\left(u_{x x}, u_{x}\right) u_{x x} \\
& =\mathcal{I}\left(u_{x x x}\right)+\left(u_{x x} \cdot u_{x x}, 0\right) \otimes(0,1)+(0,1) \otimes\left(u_{x x} \cdot u_{x x}, 0\right)
\end{aligned}
$$

and then

$$
\begin{aligned}
\partial_{n} c \cdot\left(F\left(u_{x x}, u_{x}\right)\right)_{x} & =\mathcal{I}\left(u_{x x x} \cdot \partial_{n} c\right)+K^{2} \partial_{n} c \otimes n+n \otimes K^{2} \cdot \partial_{n} c \\
& =\mathcal{I}\left(-\partial_{n} c \cdot n_{x x}\right)+K^{2} \partial_{n} c \otimes n+n \otimes K^{2} \cdot \partial_{n} c=-C .
\end{aligned}
$$

This shows that

$$
D_{t} \tilde{K}(0,0,0)=a_{K}-\partial_{n} c \cdot \partial_{y} \tilde{K}
$$

and ends the proof of the lemma.

LEMma 5.5 Let $\psi \in C^{1}\left(\mathbb{R}^{N}\right)$ and $V \in\left(C^{1}\left(\mathbb{R}^{N}\right)\right)^{N}$. Then

$$
-\int_{\Gamma_{t}} V \cdot(I-n \otimes n) \cdot \partial_{y} \psi=\int_{\Gamma_{t}} \psi\left\{(I-n \otimes n) \cdot \partial_{y}\right\} \cdot V+\int_{\Gamma_{t}} \psi(n \cdot V)(I: K) .
$$

Proof. For $\varepsilon>0$ we introduce

$$
\Omega_{\varepsilon}=\left\{x \in \mathbb{R}^{N}: \exists y \in \Gamma_{t}, x=y+r n(t, y) \text { for some } r \in(-\varepsilon, \varepsilon)\right\} .
$$

For $\varepsilon>0$ small enough and $x \in \Omega_{\varepsilon}$, there exists a unique $y=y(x)$ such that $y \in \Gamma_{t}$ and $x=y+r n(t, y)$. Then we can extend the field $n$ on $\Omega_{\varepsilon}$ by

$$
\tilde{n}(x)=n(t, y(x)) \text {. }
$$

Then

$$
\int_{\Gamma_{t}} V \cdot(I-n \otimes n) \cdot \partial_{y} \psi=\lim _{\varepsilon \rightarrow 0} \frac{1}{2 \varepsilon} \int_{\Omega_{\varepsilon}} V \cdot(I-\tilde{n} \otimes \tilde{n}) \cdot \partial_{y} \psi
$$

and

$$
-\int_{\Omega_{\varepsilon}} V \cdot(I-\tilde{n} \otimes \tilde{n}) \cdot \partial_{y} \psi=\int_{\Omega_{\varepsilon}} \psi\left\{(I-\tilde{n} \otimes \tilde{n}) \cdot \partial_{y}\right\} \cdot V-\int_{\Omega_{\varepsilon}} \psi V \cdot\left\{\left(\partial_{y} \cdot \tilde{n}\right) \tilde{n}+\left(\tilde{n} \cdot \partial_{y}\right) \tilde{n}\right\} .
$$

Hence

$$
-\int_{\Gamma_{t}} V \cdot(I-n \otimes n) \cdot \partial_{y} \psi=\int_{\Gamma_{t}} \psi\left\{(I-n \otimes n) \cdot \partial_{y}\right\} \cdot V-\int_{\Gamma_{t}} \psi V \cdot\left\{\left(\partial_{y} \cdot \tilde{n}\right) \tilde{n}+\left(\tilde{n} \cdot \partial_{y}\right) \tilde{n}\right\} .
$$

For our choice of the extension $\tilde{n}$, we have $\left(\tilde{n} \cdot \partial_{y}\right) \tilde{n}=0$, and in the coordinates $\left(\tilde{n}, \tilde{n}^{\perp}\right)$ we see from 5.35 that $\partial_{y} \cdot \tilde{n}=-I: K$, which implies the result. 
LEMMA 5.6 With the notation of Lemma 5.2, we have

$$
\int_{\Gamma_{t}} \partial_{n} c \cdot \partial_{y} \tilde{K}: \partial_{K} \varphi=\int_{\Gamma_{t}} D_{\tau} \cdot\left(\varphi \partial_{n} c\right) \quad \text { with } \quad D_{\tau}=-(I-n \otimes n) \cdot \partial_{y}+K \cdot \partial_{n}
$$

where the quantities in the integrands are evaluated at $(t, y), n(t, y), K(t, y)$.

Proof. For a general smooth function $G$, we define $\tilde{G}(t, y):=G(t, y, \tilde{n}(t, y), \tilde{K}(t, y))$. Then on $\Gamma$ we have

$$
(I-n \otimes n) \cdot \partial_{y} \tilde{G}(t, y)=\left\{(I-n \otimes n) \cdot \partial_{y}-K \cdot \partial_{n}+(I-n \otimes n) \cdot \partial_{y} \tilde{K}: \partial_{K}\right\} G
$$

where in particular we have used (5.35). We deduce that

$$
\begin{aligned}
\int_{\Gamma_{t}} \partial_{n} c \cdot \partial_{y} \tilde{K}: \partial_{K} \varphi & =\int_{\Gamma_{t}} \partial_{n} c \cdot\left\{(I-n \otimes n) \cdot \partial_{y} \tilde{K}: \partial_{K} \varphi-K \cdot \partial_{n} \varphi+(I-n \otimes n) \cdot \partial_{y} \varphi+D_{\tau} \varphi\right\} \\
& =\int_{\Gamma_{t}} \partial_{n} c \cdot(I-n \otimes n) \cdot \partial_{y} \tilde{\varphi}+\int_{\Gamma_{t}} \partial_{n} c \cdot D_{\tau} \varphi \\
& =\int_{\Gamma_{t}}\left\{-\varphi\left((I-n \otimes n) \cdot \partial_{y}\right) \cdot \widetilde{\partial_{n}} c+\partial_{n} c \cdot D_{\tau} \varphi\right\} \\
& =\int_{\Gamma_{t}}\left\{\varphi D_{\tau} \cdot \partial_{n} c+\partial_{n} c \cdot D_{\tau} \varphi\right\}=\int_{\Gamma_{t}} D_{\tau} \cdot\left(\varphi \partial_{n} c\right)
\end{aligned}
$$

where we have used 5.38 with $G=\varphi$ in the second line, Lemma 5.5 in the third line, and 5.38) with $G=\partial_{n} c$ in the fourth line. This ends the proof of the lemma.

LEMMA 5.7 For a general solution $g$ of (4.28) and $\alpha:=K \cdot n g$, we have

$$
\alpha_{t}+\partial_{y} \cdot\left(a_{y} \otimes \alpha\right)+\partial_{n} \cdot\left(a_{n} \otimes \alpha\right)+\partial_{K}:\left(a_{K} \otimes \alpha\right)+A_{0} \cdot \alpha=0
$$

with $A_{0}$ given in 4.32 .

Proof. We compute

$$
\begin{aligned}
-(K \cdot n g)_{t}= & -K \cdot n g_{t} \\
= & K \cdot n\left\{a_{0} g+\partial_{y} \cdot\left(a_{y} g\right)+\partial_{n} \cdot\left(a_{n} g\right)+\partial_{K}:\left(a_{K} g\right)\right\} \\
= & a_{0} K \cdot n g+\partial_{y} \cdot\left(a_{y} \otimes K \cdot n g\right)+\partial_{n} \cdot\left(a_{n} \otimes K \cdot n g\right)+\partial_{K}:\left(a_{K} \otimes K \cdot n g\right) \\
& -\left(a_{n} g \cdot \partial_{n}\right)(K \cdot n)-\left(a_{K} g: \partial_{K}\right)(K \cdot n),
\end{aligned}
$$

i.e. $\alpha:=K \cdot n g$ satisfies

$$
\begin{aligned}
& \alpha_{t}+a_{0} \alpha+\partial_{y} \cdot\left(a_{y} \otimes \alpha\right)+\partial_{n} \cdot\left(a_{n} \otimes \alpha\right)+\partial_{K}:\left(a_{K} \otimes \alpha\right) \\
& \quad=\left(a_{n} g \cdot \partial_{n}\right)(K \cdot n)+\left(a_{K} g: \partial_{K}\right)(K \cdot n)=K \cdot a_{n} g+a_{K} \cdot n g=-\left(A_{0}-a_{0} I\right) \cdot \alpha
\end{aligned}
$$

with

$$
-\left(A_{0}-a_{0} I\right)=c K+K \cdot \partial_{n n}^{2} c-(I-n \otimes n) \cdot \partial_{y n}^{2} c+n \otimes \partial_{y} c+2\left(n \cdot \partial_{y} c\right) I .
$$

This ends the proof of the lemma. 
Proof of Theorem 4.1. The proof is completely similar to the one of Theorem 2.1. Putting together the results of Lemmata 5.2, 5.4 and 5.6, for $g=g_{\Gamma}$ we get

$$
\begin{aligned}
\left\langle g_{t}, \varphi\right\rangle & =\left\langle g,-c(I: K) \varphi+c n \cdot \partial_{y} \varphi+D_{\tau} c \cdot \partial_{n} \varphi+a_{K}: \partial_{K} \varphi-\partial_{n} c \cdot \partial_{y} \tilde{K}: \partial_{K} \varphi\right\rangle \\
& =\left\langle g,-c(I: K) \varphi+c n \cdot \partial_{y} \varphi+D_{\tau} c \cdot \partial_{n} \varphi+a_{K}: \partial_{K} \varphi-\partial_{n} c \cdot D_{\tau} \varphi-\varphi D_{\tau} \cdot \partial_{n} c\right\rangle \\
& =\left\langle g,-a_{0} \varphi+a_{y} \partial_{y} \varphi+a_{n} \partial_{n} \varphi+a_{K} \partial_{K} \varphi\right\rangle,
\end{aligned}
$$

which leads to equation 4.28. Equation 5.39) is exactly 4.31. Finally, still for $g_{\Gamma}$ defined in 4.26 and $\bar{\psi}(t, y, n, K)=\psi(t, y, n)$,

$$
\begin{aligned}
\left\langle\hat{g}_{\Gamma}, \psi\right\rangle & =\left\langle D_{\tau} g+n(I: K) g, \bar{\psi}\right\rangle \\
& =\left\langle g, n(I: K) \bar{\psi}-D_{\tau} \bar{\psi}\right\rangle \\
& =\left\langle g, n(I: K) \bar{\psi}+(I-n \otimes n) \cdot \partial_{y} \bar{\psi}-K \cdot \partial_{n} \bar{\psi}+(I-n \otimes n) \cdot \partial_{y} \tilde{K}: \partial_{K} \bar{\psi}\right\rangle \\
& =\int_{0}^{T} \mathrm{~d} t \int_{\Gamma_{t}}\left\{n(I: K) \tilde{\psi}(t, y)+(I-n \otimes n) \cdot \partial_{y} \tilde{\psi}(t, y)\right\}=0
\end{aligned}
$$

where in the third line we have used the fact that $\bar{\psi}$ is independent of the curvature $K$, and in the fourth line we have used equation 5.38 with $G=\bar{\psi}$ and $\tilde{\psi}(t, y)=\bar{\psi}(t, y, n(t, y), K(t, y))$. For the last line, we have used Lemma 5.5 with $V=e_{i}, i=1, \ldots, N$, succesively. This proves that $\hat{g}_{\Gamma}=0$, and ends the proof of the theorem.

REMARK 5.8 We remark that if $K \cdot n g=0$, then $(I-n \otimes n) \cdot K \cdot \partial_{n} g=K \cdot \partial_{n} g+(I: K) n g$. This last relation explains why the contribution of the term $n(I: K)$ to the definition of $\hat{g}_{\Gamma}$ does not vanish in general, even if $\hat{g}_{\Gamma}=0$.

\section{Appendix}

\subsection{Proof of Lemma 5.1}

We consider a point $\left(t_{0}, y_{0}\right) \in \Gamma$, and a local parametrization $\gamma: B_{r_{0}} \rightarrow \mathbb{R}^{N}$ of $\Gamma_{t_{0}}$ in a neighbourhood of $y_{0}$, with $B_{r_{0}}=B_{r_{0}}(0) \subset \mathbb{R}^{N-1}$ and $r_{0}>0$. Then, in a neighbourhood $U_{t_{0}, y_{0}}$, we can parametrize $\Gamma_{t_{0}+h}$, for $h$ small, by $\gamma^{t_{0}+h}$ defined by

$$
\gamma^{t_{0}+h}(x)=\gamma(x)+r(h, x) n(x)
$$

where $n(x)$ is the normal $n$ to $\gamma$ at the point $\gamma(x)$.

We will prove that formula 5.33 holds, assuming moreover that

$$
\operatorname{supp} \psi \subset U_{t_{0}, y_{0}} .
$$

Finally, using a partition of unity, we obtain the full formula (5.33) as in the lemma.

We now prove (5.33) assuming 6.40). We have $r(0, x)=0$ and $r_{h}(0, x)=c(t, \gamma(x))$ (where $r_{h}$ stands for $\partial r / \partial h, r_{i}$ stands for $\partial r / \partial x_{i}$, and $r_{i h}$ stands for $\left.\partial^{2} r / \partial x_{i} \partial h\right)$. We compute the jacobian

$$
J_{h}=\left|a_{h}\right| \quad \text { with }\left\langle a_{h}, \cdot\right\rangle=\operatorname{det}\left(\gamma_{1}^{t_{0}+h}, \ldots, \gamma_{N-1}^{t_{0}+h}, \cdot\right)
$$

where we have set

$$
\gamma_{i}^{t_{0}+h}=\frac{\partial \gamma^{t_{0}+h}}{\partial x_{i}}=\gamma_{i}+r_{i} n+r \frac{\partial n}{\partial x_{i}}
$$


and similarly $\gamma_{i}=\partial \gamma / \partial x_{i}$ for $i=1, \ldots, N-1$. We have

$$
\int_{\Gamma_{T_{0}+h}} \psi\left(t_{0}+h, \cdot\right)=\int_{B_{r_{0}}} \mathrm{~d} x \psi\left(t_{0}+h, \gamma^{t_{0}+h}(x)\right) J_{h}=\int_{B_{r_{0}}} \mathrm{~d} x \psi(t+h, \gamma(x)+r(h, x) n) J_{h} .
$$

Therefore

$$
\frac{\mathrm{d}}{\mathrm{d} h}\left(\int_{\Gamma_{t_{0}+h}} \psi(t+h, \cdot)\right)_{\mid h=0}=\int_{B_{r_{0}}} \mathrm{~d} x\left\{\left(\psi_{t}+r_{h}(0, x) n \cdot \partial_{y} \psi\right) J_{0}+\psi J_{0}^{\prime}\right\}
$$

with

$$
J_{0}^{\prime}=\left(\frac{\mathrm{d} J_{h}}{\mathrm{~d} h}\right)_{\mid h=0}=\frac{a_{0} \cdot a_{0}^{\prime}}{\left|a_{0}\right|}
$$

with $a_{0}^{\prime}=\left(\mathrm{d} a_{h} / \mathrm{d} h\right)_{\mid h=0}$. On the other hand, we have

$$
\left|a_{h}\right|^{2}=\operatorname{det}\left(\gamma_{1}^{t_{0}+h}, \ldots, \gamma_{N-1}^{t_{0}+h}, a_{h}\right)
$$

which by differentiation gives

$$
2 a_{0} \cdot a_{0}^{\prime}=\operatorname{det}\left(\gamma_{1}, \ldots, \gamma_{N-1}, a_{0}^{\prime}\right)+\sum_{i=1}^{N-1} \operatorname{det}\left(\gamma_{1}, \ldots, \gamma_{i}^{t_{0}{ }^{\prime}}, \ldots, \gamma_{N-1}, a_{0}\right)
$$

with (from 6.40 )

$$
\gamma_{i}^{t_{0}^{\prime}}=\left(\frac{\partial \gamma_{i}^{t_{0}+h}}{\partial h}\right)_{\mid h=0}=r_{i h}(0, x) n+r_{h}(0, x) \frac{\partial n}{\partial x_{i}} .
$$

Therefore

$$
a_{0} \cdot a_{0}^{\prime}=\sum_{i=1}^{N-1} \operatorname{det}\left(\gamma_{1}, \ldots, r_{i h} n+r_{h} \frac{\partial n}{\partial x_{i}}, \ldots, \gamma_{N-1}, a_{0}\right) .
$$

Using in particular the fact that $a_{0}$ is parallel to $n$, we deduce that

$$
a_{0} \cdot a_{0}^{\prime}=r_{h}(0, x)\left|a_{0}\right|^{2} k \quad \text { with } \quad k:=\sum_{i=1}^{N-1} \frac{\left\langle\gamma_{i}, \partial n / \partial x_{i}\right\rangle}{\left|\gamma_{i}\right|^{2}} .
$$

A direct computation in local coordinates shows that $k=-I: K$, which gives

$$
J_{0}^{\prime}=-c(I: K) J_{0} .
$$

Finally, 6.42) implies

$$
\begin{aligned}
\frac{\mathrm{d}}{\mathrm{d} h}\left(\int_{\Gamma_{t_{0}+h}} \psi(t+h, \cdot)\right)_{\mid h=0} & =\int_{B_{r_{0}}} \mathrm{~d} x J_{0}\left\{\psi_{t}+c n \cdot \partial_{y} \psi-c(I: K) \psi\right\} \\
& =\int_{\Gamma_{t_{0}}}\left\{\psi_{t}+c n \cdot \partial_{y} \psi-c(I: K) \psi\right\} .
\end{aligned}
$$

This ends the proof of the lemma. 


\subsection{Case of a curve transported in dimension $\bar{N}$}

In this subsection, we consider a curve $\bar{\Gamma}_{t}$ transported in $\mathbb{R}^{\bar{N}}$ along a vector field $\bar{c}(t, \bar{y}) \in \mathbb{R}^{\bar{N}}$, in dimension $\bar{N} \geqslant 2$. Here we use the notation $\bar{N}$ rather than $N$ for the dimension, because $\bar{N}$ can be different from $N$, as in the application given in Subsection 6.3. We also emphasize that we do not consider the case of "normal velocity", possibly depending on the unit tangent vector to the curve $\bar{\Gamma}_{t}$, which we denote by $\bar{\tau} \in \mathbb{S}^{\bar{N}-1}$.

Let $\bar{\kappa} \in \mathbb{R}^{\bar{N}}$ be the curvature vector of the curve, $\bar{\kappa}=\mathrm{d} \bar{\tau} / \mathrm{d} s$, and set

$$
g_{\bar{\Gamma}}(t, \bar{y}, \bar{\tau}, \bar{\kappa})=\delta_{\bar{\Gamma}_{t}}(\bar{y}) \delta_{0}(\bar{\tau}-\bar{\tau}(t, \bar{y})) \delta_{0}(\bar{\kappa}-\bar{\kappa}(t, \bar{y})) .
$$

Then we have:

PRoposition 6.1 (Linear transport equation in the phase space for geometric transport of a curve in $\mathbb{R}^{\bar{N}}$ ) Assume that a smooth curve $\bar{\Gamma}_{t}$ is transported in $\mathbb{R}^{\bar{N}}$ along a smooth vector field $\bar{c}(t, \bar{y}) \in \mathbb{R}^{\bar{N}}$. Then the distribution $g=g_{\bar{\Gamma}}$ defined in 6.43 satisfies

$$
g_{t}+\operatorname{div}(\bar{a} g)+\bar{a}_{0} g=0
$$

with

$$
\left\{\begin{array}{l}
\bar{a}_{0}=\bar{c} \cdot \bar{\kappa}, \quad \bar{a}_{\bar{y}}=(I-\bar{\tau} \otimes \bar{\tau}) \cdot \bar{c}, \quad \bar{a}_{\bar{\tau}}=(I-\bar{\tau} \otimes \bar{\tau}) \cdot\left(\bar{\tau} \cdot \partial_{\bar{y}} \bar{c}\right), \\
\bar{a}_{\bar{\kappa}}=(I-\bar{\tau} \otimes \bar{\tau}) \cdot\left\{\bar{\kappa} \cdot \partial_{\bar{y}} \bar{c}+\bar{\tau} \otimes \bar{\tau}: \partial_{\bar{y} y} \frac{2}{c}\right\}-(\bar{\kappa} \otimes \bar{\tau}+\bar{\tau} \otimes \bar{\kappa}) \cdot\left(\bar{\tau} \cdot \partial_{\bar{y}} \bar{c}\right) .
\end{array}\right.
$$

The proof of Proposition 6.1 uses the following result (analogous to Lemma 5.1):

LEMMA 6.2 For a smooth curve $\bar{\Gamma}_{t}$ transported in $\mathbb{R}^{\bar{N}}$ along a smooth vector field $\bar{c}(t, \bar{y}, \bar{\tau}) \in \mathbb{R}^{\bar{N}}$ (which is not assumed to be perpendicular to $\bar{\tau}$ ), for any smooth function $\psi$ we have

$$
\frac{\mathrm{d}}{\mathrm{d} t}\left(\int_{\bar{\Gamma}_{t}} \psi\right)=\int_{\bar{\Gamma}_{t}} D_{t} \psi-\bar{c} \cdot \bar{\kappa} \psi \quad \text { with } \quad D_{t} \psi=\psi_{t}+\bar{c} \cdot(I-\bar{\tau} \otimes \bar{\tau}) \cdot \partial_{\bar{y}} \psi
$$

Sketch of proof of Proposition 6.1. To get equation 6.44, we simply follow the lines of our approach for hypersurfaces. For a general vector field $\bar{c}(t, \bar{y}, \bar{\tau})$ (which here depends on $\bar{\tau}$ ), we get

$$
\left\langle g_{t}, \varphi\right\rangle=\left\langle g,-\bar{c} \cdot \bar{\kappa} \varphi+\bar{c} \cdot(I-\bar{\tau} \otimes \bar{\tau}) \cdot \partial_{\bar{y}} \varphi+D_{t} \tilde{\bar{\tau}} \cdot \partial_{\bar{\tau}} \varphi+D_{t} \tilde{\bar{\kappa}} \cdot \partial_{\bar{\kappa}} \varphi\right\rangle
$$

with

$$
\left\{\begin{aligned}
D_{t} \tilde{\bar{\tau}}= & D_{\tau} \bar{c} \quad \text { with } \quad D_{\tau}=(I-\bar{\tau} \otimes \bar{\tau}) \cdot\left(\bar{\tau} \cdot \partial_{\bar{y}}+\bar{\kappa} \cdot \partial_{\bar{\tau}}\right), \\
D_{t} \tilde{\bar{\kappa}}= & a_{\bar{\kappa}}+(I-\bar{\tau} \otimes \bar{\tau}) \cdot\left(\frac{\mathrm{d} \bar{\kappa}}{\mathrm{d} s} \cdot \partial_{\bar{\tau}} \bar{c}\right), \\
a_{\bar{\kappa}}= & (I-\bar{\tau} \otimes \bar{\tau}) \cdot\left\{\bar{\kappa} \cdot \partial_{\bar{y}} \bar{c}+\bar{\tau} \otimes \bar{\tau}: \partial_{\overline{y y}} \bar{c}+2 \bar{\tau} \cdot \partial_{\bar{y} \bar{\tau}} \bar{c} \cdot \bar{\kappa}+\bar{\kappa} \otimes \bar{\kappa}: \partial_{\bar{\tau} \tau} \frac{2}{\bar{c}}\right\} \\
& -(\bar{\kappa} \otimes \bar{\tau}+\bar{\tau} \otimes \bar{\kappa}) \cdot\left(\bar{\tau} \cdot \partial_{\bar{y}} \bar{c}+\bar{\kappa} \cdot \partial_{\bar{\tau}} \bar{c}\right) .
\end{aligned}\right.
$$

Indeed, we see that in the expression of $\left\langle g_{t}, \varphi\right\rangle$, there is a term

$$
(I-\bar{\tau} \otimes \bar{\tau}) \cdot\left(\frac{\mathrm{d} \bar{\kappa}}{\mathrm{d} s} \cdot \partial_{\bar{\tau}} \bar{c}\right) \cdot \partial_{\bar{\kappa}} \varphi
$$


where $\partial_{\bar{\tau}} \bar{c}$ is a matrix. Because of this new term, we cannot use the trick applied in the case of hypersurfaces, where we rewrote this term, essentially as $\partial_{\bar{\tau}} \bar{c}$ times $(\mathrm{d} \bar{\kappa} / \mathrm{d} s) \cdot \partial_{\bar{\kappa}} \varphi$, and then concluded by an integration by parts.

Nevertheless, in the case where the vector field $\bar{c}$ is independent of $\bar{\tau}$, we easily find that $g$ solves equation 6.44.

\subsection{Application to another transport formulation for a curve moving in the plane}

Let us remark that the formalism of Subsection 6.2 can be applied to the particular case of a curve $\Gamma_{t}$ moving in the plane with a normal velocity $c(t, y, \theta)$, to which we associate the corresponding curve in the space/angle coordinates

$$
\bar{\Gamma}_{t}=\left\{\begin{array}{l}
\bar{y}=(y, \theta(t, y)) \in \mathbb{R}^{2} \times(\mathbb{R} /(2 \pi \mathbb{Z})) \\
\text { for } y \in \Gamma_{t} \text { with unit tangent vector } \tau(t, y)=(\sin \theta(t, y),-\cos \theta(t, y))
\end{array}\right\} .
$$

The curve $\bar{\Gamma}_{t}$ moves with velocity

$$
\bar{c}(t, \bar{y})=a^{\prime}(t, y, \theta) \quad \text { with } \quad \bar{y}=(y, \theta) \in \mathbb{R}^{2} \times(\mathbb{R} /(2 \pi \mathbb{Z}))
$$

where $a^{\prime}=\left(a_{y}, a_{\theta}\right)$ is defined in Theorem 2.1 $(N=2)$. Here this velocity does not depend on $\bar{\tau}$, the unit tangent vector to $\bar{\Gamma}_{t}$, which is given by

$$
\bar{\tau}(t, \bar{y})=\frac{1}{\sqrt{1+\kappa^{2}}}(\tau, \kappa)
$$

where $\kappa(t, y)$ is the (scalar) curvature of the original curve $\Gamma_{t}$.

Applying now Proposition 6.1 with $\bar{N}=3$, we get the following result:

COROLlaRY 6.3 (A transport equation associated to a curve, with at most linear growth in its coefficients) For the curve $\bar{\Gamma}_{t}$ defined in $\sqrt{6.46}$, the distribution $g_{\bar{T}}$ satisfies equation 6.44 with coefficients $\bar{a}, \bar{a}_{0}$ given in 6.45 where $\bar{c}$ is defined in 6.47 with $\bar{N}=3$. Moreover, the vector field $\bar{a}$ has at most linear growth in the curvature vector $\bar{\kappa}$ defined in Subsection 6.2

The advantage here to consider $\bar{\Gamma}_{t}$ in place of $\Gamma_{t}$ is that the curve $\bar{\Gamma}_{t}$ stays regular for all time (this is the wavefront; see for instance Osher et al. [25] for the wavefront associated to curves and Leung, Qian and Osher [20] for the wavefront associated to surfaces), while $\Gamma_{t}$ can become singular in finite time. At the level of the transport equation 6.44 satisfied by $g_{\bar{\Gamma}}$ in Corollary 6.3, the nice property is that the vector field $\bar{a}$ has at most linear growth in $\bar{\kappa}$. A consequence is the existence of solutions of (6.44) for all time in that case.

REMARK 6.4 From the previous point of view, it is natural to ask if there are some relations between first order evolution of curves in $\mathbb{R}^{3}$ and second order evolution of curves in the plane.

REMARK 6.5 Similarly, it could be interesting for hypersurfaces $\Gamma_{t}$ to consider

$$
\bar{\Gamma}_{t}=\left\{\begin{array}{l}
\bar{y}=(y, n(t, y)) \in \mathbb{R}^{N} \times \mathbb{S}^{N-1} \\
\text { for } y \in \Gamma_{t} \text { with unit normal vector } n(t, y)
\end{array}\right\}
$$


and to try to write a transport equation for the measure

$$
g_{\bar{\Gamma}}(t, \bar{y}, \bar{n}, \bar{K})=\delta_{\bar{\Gamma}_{t}}(\bar{y}) \delta_{0}(\bar{n}-\bar{n}(t, \bar{y})) \delta_{0}(\bar{K}-\bar{K}(t, \bar{y}))
$$

where $\bar{n}(t, \bar{y})$ defines the tangent space (of dimension $N-1$ ) to $\bar{\Gamma}_{t}$ at $\bar{y}$, and $\bar{K}(t, \bar{y}$ ) defines its curvature. It would also be interesting to see how to extend this method for the evolution of general submanifolds of arbitrary codimension.

\section{Acknowledgements}

I would like to thank Radan Sedláček for enlightening discussions which motivated this work and T. Hochrainer for providing Remark 2.3. We also thank the unknown referees for their suggestions improving the presentation of the manuscript.

\section{REFERENCES}

1. Alvarez, O., Cardaliaguet, P., \& Monneau, R. Existence and uniqueness for dislocation dynamics with nonnegative velocity. Interfaces Free Bound. 7 (2005), 415-434. Zbl 1099.35148 MR 2191694

2. Alvarez, O., Hoch, P., Le Bouar, Y., \& Monneau, R. Dislocation dynamics: short time existence and uniqueness of the solution. Arch. Ration. Mech. Anal. 181 (2006), 449-504. Zbl pre05051264 MR 2231781

3. Buckdahn, R., Cardaliaguet, P., \& Quincampoix, M. A representation formula for the mean curvature motion. SIAM J. Math. Anal. 33 (2001), 827-846. Zbl 1074.93037| MR 884724

4. Cannarsa, P., \& Cardaliaguet, P. Perimeter estimates for reachable sets of control systems. J. Convex Anal. 13 (2006), 253-267. MR 2252231

5. CANNARSA, P., \& FRANKOWSKA, H. Interior sphere property of attainable sets and the time optimal control problems. ESAIM Control Optim. Calc. Var. 12 (2006), 350-370. Zbl 1105.93007 MR 2209357

6. El-Azab, A. The boundary value problem of dislocation dynamics. Model. Simul. Mater. Sci. Engrg. 8 (2000), 37-54.

7. EL-AZAB, A. Statistical mechanics treatment of the evolution of dislocation distributions in single crystals. Phys. Rev. B 61 (2000), 11956-11966.

8. El HajJ, A., \& ForCADEl, N. A convergent scheme for a non-local coupled system modelling dislocations densities dynamics. Math. Comp., to appear.

9. Evans, L. C. Partial Differential Equations. Grad. Stud. in Math. 19, Amer. Math. Soc., Providence, RI (1998). Zbl 0902.35002 MR 1625845

10. Groma, I., \& BALOGH, P. Link between the individual and continuum approaches of the description of the collective behaviour of dislocations. Mater. Sci. Engrg. A 234-236 (1997), 249-252.

11. GRoma, I., \& BALOGH, P. Investigation of dislocations pattern formation in a two-dimensional selfconsistent field approximation. Acta Mater. 47 (1999), 3647-3654.

12. Hochrainer, T. A continuum theory of dislocation motion. Proc. 2nd Int. Conf. on Multiscale Materials Modeling (Los Angeles, 2004), N. M. Ghoniem (ed.), Univ. of California, Los Angeles (2004), 210-212.

13. Hochrainer, T., \& Zaiser, M. Fundamentals of a continuum theory of dislocations. Statistical Mechanics of Plasticity and Related Instabilities (SOMPRI 2005, Bangalore), M. Zaiser et al. (eds.), to appear.

14. Hochrainer, T., Zaiser, M., \& Gumbsch, P. A three-dimensional continuum theory of dislocation systems: kinematics and mean-field formulation. Philos. Mag. 87 (2007), 1261-1282. 
15. Imbert, C., \& Monneau, R. Homogenization of first order equations with $(u / \varepsilon)$-periodic Hamiltonians. Part II: application to dislocations dynamics. Arch. Ration. Mech. Anal., to appear.

16. Kosevich, A. M. Dynamical theory of dislocations. Soviet Physics Uspekhi 7 (1965), 837-854. MR 182214

17. KRÖNER, E. Kontinuumstheorie der Versetzungen und Eigenspannungen. Ergeb. Angew. Math. 5, Springer, Berlin (1958). Zbl 0084.40003 MR 0095615

18. KRÖNER, E. Benefit and shortcomings of the continuous theory of dislocations. Internat. J. Solids Structures 38 (2001), 1115-1134. Zbl 1010.74011

19. Lardner, R. W. Mathematical Theory of Dislocations and Fracture. Math. Expo. 17, Univ. Toronto Press (1974). Zbl 0301.73036 MR 371203

20. Leung, S., Qian, J., \& Osher, S. A level set method for three-dimensional paraxial geometrical optics with multiple point sources. Comm. Math. Sci. 2 (2004), 643-672. Zbl 02247552 MR 2119932

21. LEVEQUE, R. J. Numerical Methods for Conservation Laws. Lectures in Math. ETH Zürich, Birkhäuser, Basel (1990). Zbl 0723.65067 MR 1077828

22. Lions, P.-L. Generalized Solutions of Hamilton-Jacobi Equations, Res. Notes Math. 69, Pitman, Boston, MA (1982). Zbl 0497.35001 MR 667669

23. Mura, T. Continuous distribution of moving dislocations. Philos. Mag. 8 (1963), 843-857.

24. Oseledets, V. I. On a new way of writing the Navier-Stokes equation: the Hamiltonian formalism. Uspekhi Mat. Nauk 44 (1989), no. 3, 169-170 (in Russian); English transl.: Russian Math. Surveys 44 (1989), 210-211. Zbl 0850.76130 MR 1024057

25. Osher, S., Cheng, L.-T., KAng, M., Shim, H., \& Tsai, Y.-H. Geometric optics in a phase-spacebased level set and Eulerian framework. J. Comput. Phys. 179 (2002), 622-648. Zbl 0999.78002 MR 1911378

26. Perthame, B. Kinetic Formulation of Conservation Laws. Oxford Univ. Press (2002). Zbl 1030.35002 MR 2064166

27. SedláčeK, R., KRATOChvíl, J., \& Werner, E. The importance of being curved: bowing dislocations in a continuum description. Philos. Mag. 83 (2003), 3735-3752.

28. SONER, H. M., \& TOUZI, N. Dynamics programming for stochastic target problems and geometric flows. J. Eur. Math. Soc. 4 (2002), 201-236. Zbl 1003.49003 MR 1924400

29. Soner, H. M., \& TouzI, N. Level set characterization of stochastic target problems. Comm. Partial Differential Equations 27 (2002), 2031-2053. MR 1941665 MR 1988466

30. Soner, H. M., \& TouZI, N. A stochastic representation for mean curvature type geometric flows. Ann. Probab. 31 (2003), 1145-1165. Zbl 1080.60076

31. Zaiser, M., \& Hochrainer, T. Some steps towards a continuum representation of $3 \mathrm{~d}$ dislocation systems. Scripta Mater. 54 (2005), 717-721. 